\title{
Substituent Effects on the Z/E-Selectivity in Cross-Metathesis of Conjugated Enynes
}

\author{
Byungman Kang, Ji Min Lee, Jaesik Kwak, Yoon Sup Lee* and Sukbok Chang* \\ Center for Molecular Design and Synthesis (CMDS), Department of Chemistry and School of Molecular Science \\ (BK21), Korea Advanced Institute of Science and Technology, Daejeon 305-701, Korea
}

\section{Supporting Information}

\section{Table of Contents}

General S3

Representative experimental procedure of enyne metathesis.

Experimental Procedure of CM of 4-hexen-2-ynyl benzoate and allyl trimetylsilane with Catalyst 3.

${ }^{1} \mathrm{H}$ NMR, ${ }^{13} \mathrm{C}$ NMR and HRMS (EI) of [(Z)-4-Hepten-2-ynyl] benzyl ether (1) S4

${ }^{1} \mathrm{H} N M R,{ }^{13} \mathrm{C}$ NMR and HRMS (EI) of [(Z)-6-Nonen-4-ynyl] benzyl ether (2) S4

${ }^{1} \mathrm{H} N M R,{ }^{13} \mathrm{C}$ NMR and HRMS (EI) of [(Z)-12-pentadecen-10-ynyl] benzyl ether (3) S5

${ }^{1} \mathrm{H} N M R,{ }^{13} \mathrm{C}$ NMR and HRMS (EI) of [(Z)-6-Trimethylsilyl-4-hexen-2-ynyl] benzyl ether (4) S5

${ }^{1} \mathrm{H} N M R,{ }^{13} \mathrm{C}$ NMR and HRMS (EI) of [(Z)-8-Trimethylsilyl-6-octen-4-ynyl] benzyl ether (5) S6

${ }^{1} \mathrm{H}$ NMR, ${ }^{13} \mathrm{C}$ NMR and HRMS (EI) of [(Z)-4-Hepten-2-ynyl] benzoate (6) S6

${ }^{1} \mathrm{H} N M R,{ }^{13} \mathrm{C}$ NMR and HRMS (EI) of [(Z)-6-Nonen-4-ynyl] benzoate (7) S6

${ }^{1} \mathrm{H} N M R,{ }^{13} \mathrm{C}$ NMR and HRMS (EI) of [(Z)-12-Pentadecen-10-ynyl] benzoate (8) S7

${ }^{1} \mathrm{H}$ NMR, ${ }^{13} \mathrm{C}$ NMR and HRMS (EI) of [(Z)-6-Trimethylsilyl-4-hexen-2-ynyl] benzoate (9) S7

${ }^{1} \mathrm{H}$ NMR, ${ }^{13} \mathrm{C}$ NMR and HRMS (EI) of [(Z)-8-Trimethylsilyl-6-octen-4-ynyl] benzoate (10) $\quad$ S7

${ }^{1} \mathrm{H}$ NMR, ${ }^{13} \mathrm{C}$ NMR and HRMS (EI) of $N$-Ethyl- $N$-[(Z)-4-hepten-2-ynyl] benzamide (11) S8

${ }^{1} \mathrm{H}$ NMR, ${ }^{13} \mathrm{C}$ NMR and HRMS (EI) of $N$-Ethyl- $N$-[(Z)-4-hepten-2-ynyl] $p$-toluenesulfonamide

${ }^{1} \mathrm{H}$ NMR, ${ }^{13} \mathrm{C}$ NMR and HRMS (EI) of $N$-Ethyl- $N$-[6-trimethylsilyl-(Z)-4-hexen-2-ynyl] $p$ toluenesulfonamide (13) $\quad$ S8

${ }^{1} \mathrm{H}$ NMR, ${ }^{13} \mathrm{C}$ NMR and HRMS (EI) of [(Z)-5-octen-3-ynyl] tosylate (15) S9

Calculated energies (in hartree) of chelation and non-chelation metallacyclobutane intermediates of the reaction derived from Scheme 3 in the text.

S10 
${ }^{1} \mathrm{H}$ NMR and ${ }^{13} \mathrm{C}$ NMR Spectrums of [(Z)-4-Hepten-2-ynyl] benzyl ether (1) S11

${ }^{1} \mathrm{H} N M R$ and ${ }^{13} \mathrm{C}$ NMR Spectrums of [(Z)-6-Nonen-4-ynyl] benzyl ether (2) S12

${ }^{1} \mathrm{H}$ NMR and ${ }^{13} \mathrm{C}$ NMR Spectrums of [(Z)-12-pentadecen-10-ynyl] benzyl ether (3) S13

${ }^{1} \mathrm{H}$ NMR and ${ }^{13} \mathrm{C}$ NMR Spectrums of [(Z)-6-Trimethylsilyl-4-hexen-2-ynyl] benzyl ether (4) S14

${ }^{1} \mathrm{H}$ NMR and ${ }^{13} \mathrm{C}$ NMR Spectrums of [(Z)-8-Trimethylsilyl-6-octen-4-ynyl] benzyl ether (5) S15

${ }^{1} \mathrm{H}$ NMR and ${ }^{13} \mathrm{C}$ NMR Spectrums of [(Z)-4-Hepten-2-ynyl] benzoate (6) S16

${ }^{1} \mathrm{H}$ NMR and ${ }^{13} \mathrm{C}$ NMR Spectrums of [(Z)-6-Nonen-4-ynyl] benzoate (7) S17

${ }^{1} \mathrm{H}$ NMR and ${ }^{13} \mathrm{C}$ NMR Spectrums of [(Z)-12-Pentadecen-10-ynyl] benzoate (8) S18

${ }^{1} \mathrm{H}$ NMR and ${ }^{13} \mathrm{C}$ NMR Spectrums of [(Z)-6-Trimethylsilyl-4-hexen-2-ynyl] benzoate (9) $\quad$ S19

${ }^{1} \mathrm{H}$ NMR and ${ }^{13} \mathrm{C}$ NMR Spectrums of [(Z)-8-Trimethylsilyl-6-octen-4-ynyl] benzoate (10) S20

${ }^{1} \mathrm{H}$ NMR and ${ }^{13} \mathrm{C}$ NMR Spectrums of $N$-Ethyl- $N$-[(Z)-4-hepten-2-ynyl] benzamide (11) S21

${ }^{1} \mathrm{H}$ NMR and ${ }^{13} \mathrm{C}$ NMR Spectrums of $N$-Ethyl- $N$-[(Z)-4-hepten-2-ynyl] $p$-toluenesulfonamide (12)

S22

${ }^{1} \mathrm{H}$ NMR and ${ }^{13} \mathrm{C}$ NMR Spectrums of $N$-Ethyl- $N$-[6-trimethylsilyl-(Z)-4-hexen-2-ynyl] $p$ toluenesulfonamide (13) $\quad$ S23

${ }^{1} \mathrm{H}$ NMR and ${ }^{13} \mathrm{C}$ NMR Spectrums of [(Z)-7-decen-5-ynyl] tosylate (14) S24

${ }^{1}$ H NMR and ${ }^{13} \mathrm{C}$ NMR Spectrums of [(Z)-5-octen-3-ynyl] tosylate (15) S25

Notes and References $\quad$ S26 
General. Chemical shift are reported in delta $(\delta)$ units, parts per million (ppm) downfield from tetramethylsilane, or in ppm relative to the singlet at $7.26 \mathrm{ppm}$ of chloroform- $d 1$. Splitting patterns are designated as follows: s; singlet, d; doublet, t; triplet, q; quartet, m; multiplet, td; doublet of triplet, dt; triplet of doublet, qd; doublet of quintet, b; broad. Catalyst $\left.\left(\mathrm{H}_{2} \mathrm{IMes}\right)(3-\mathrm{Br}-\mathrm{Py})_{2}(\mathrm{Cl})_{2} \mathrm{Ru}=\mathrm{CHPh}\right)$ (3) was prepared starting from $\left(\mathrm{H}_{2} \mathrm{IMes}\right)\left(\mathrm{PCy}_{3}\right)(\mathrm{Cl})_{2}$ $\mathrm{Ru}=\mathrm{CH} \mathrm{Ph}(2)$ according to the reported procedure. ${ }^{1}$

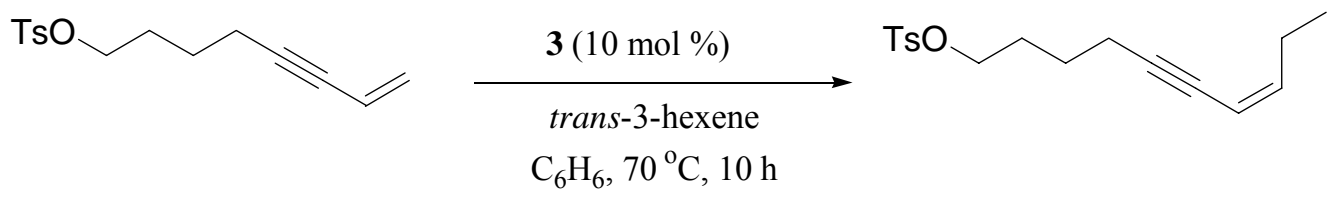

Representative experimental procedure of enyne metathesis. To a solution of 7-octen-5yne-1-yl p-toluenesulfonate $(111 \mathrm{mg}, 0.39 \mathrm{mmol})$ in benzene $(1.0 \mathrm{~mL})$ was added trans-3hexene $(0.15 \mathrm{~mL}, 1.1 \mathrm{mmol})$ followed by catalyst $3(18 \mathrm{mg}, 0.019 \mathrm{mmol})$ in benzene $(1.5 \mathrm{~mL})$. The reaction mixture was stirred for $5 \mathrm{~h}$ at $70{ }^{\circ} \mathrm{C}$ under nitrogen atmosphere. Then catalyst 3 (18 $\mathrm{mg}, 0.019 \mathrm{mmol})$ in benzene $(1.5 \mathrm{~mL})$ was added again and the mixture was stirred for another $5 \mathrm{~h}$ at the same temperature. After removal of organic solvent under reduced pressure, the residue was purified by a column chromatography on silica gel (ethyl acetate:hexane, 1/7) to afford the corresponding enyne as an analytically pure isomer, [(Z)-7-decen-5-ynyl] tosylate (14) (97 mg, $81 \%):{ }^{1} \mathrm{H}$ NMR (400 MHz, $\left.\mathrm{CDCl}_{3}\right) \delta 7.75(\mathrm{~d}, 2 \mathrm{H}, J=8.3 \mathrm{~Hz}), 7.30$ (d, $2 \mathrm{H}, J=8.2 \mathrm{~Hz}), 5.77(\mathrm{dt}, 1 \mathrm{H}, J=10.6,7.3 \mathrm{~Hz}), 5.32(\mathrm{~d}, 1 \mathrm{H}, J=10.7 \mathrm{~Hz}), 4.02(\mathrm{t}, 2 \mathrm{H}, J=6.2$ $\mathrm{Hz}), 2.41(\mathrm{~s}, 3 \mathrm{H}), 2.28(\mathrm{td}, 2 \mathrm{H}, J=6.9,2.1 \mathrm{~Hz}), 2.21(\mathrm{qd}, 2 \mathrm{H}, J=7.5,1.2 \mathrm{~Hz}), 1.71-1.78(\mathrm{~m}$, 2H), 1.49-1.56 (m, 2H), $0.95(\mathrm{t}, 3 \mathrm{H}, J=7.1 \mathrm{~Hz}) ;{ }^{13} \mathrm{C} \mathrm{NMR}\left(100 \mathrm{MHz}, \mathrm{CDCl}_{3}\right) \delta 144.7,144.3$, $132.9,129.7,127.7,108.3,92.9,77.8,69.9,27.8,24.5,23.4,21.5,18.7,13.3$; HRMS (EI) calcd for $\mathrm{C}_{17} \mathrm{H}_{22} \mathrm{O}_{3} \mathrm{~S}\left[\mathrm{M}^{+}\right]$306.1290, found 306.1292. 


\section{Experimental Procedure of CM of 4-hexen-2-ynyl benzoate and allyl trimetylsilane with}

Catalyst 3. To a solution of 4-hexen-2-ynyl benzoate $(74 \mathrm{mg}, 0.39 \mathrm{mmol})$ in benzene $(1.0$ $\mathrm{mL}$ ) was added allyl trimethylsilane $(0.15 \mathrm{~mL}, 1.1 \mathrm{mmol})$ followed by catalyst $3(18 \mathrm{mg}$, $0.019 \mathrm{mmol})$ in benzene $(1.5 \mathrm{~mL})$. The reaction mixture was stirred for $4.5 \mathrm{~h}$ at $70{ }^{\circ} \mathrm{C}$ under nitrogen atmosphere, and at $0.5,2$ and $4 \mathrm{~h}, 0.5 \mathrm{~mL}$ of reaction mixture was sampled each time and purified by short column. At $4.5 \mathrm{~h}$, catalyst $3(18 \mathrm{mg}, 0.019 \mathrm{mmol})$ in benzene $(1.5 \mathrm{~mL})$ was added and $0.5 \mathrm{~mL}$ of reaction mixture was sampled at $2 \mathrm{~h}$ interval, purified by short column to determine the conversion (\%) and $Z / E$ ratio. The conversion (\%) and $Z / E$ ratio was determined via crude ${ }^{1} \mathrm{H}$ NMR by comparing the area of vinyl proton of $Z \& E$ - products with vinyl proton of the starting material.

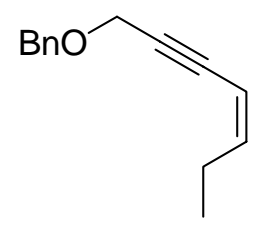

[(Z)-4-Hepten-2-ynyl] benzyl ether (1): ${ }^{1} \mathrm{H} \mathrm{NMR}\left(400 \mathrm{MHz}, \mathrm{CDCl}_{3}\right) \delta$ 7.28-7.19 (m, 5H), $5.86(\mathrm{dt}, 1 \mathrm{H}, J=10.7,7.4 \mathrm{~Hz}), 5.40(\mathrm{~d}, 1 \mathrm{H}, J=10.7 \mathrm{~Hz}), 4.54(\mathrm{~s}, 2 \mathrm{H}), 4.25(\mathrm{~d}, 2 \mathrm{H}, J=2.5$ $\mathrm{Hz}), 2.26(\mathrm{qd}, 2 \mathrm{H}, J=7.5,1.1 \mathrm{~Hz}), 0.95(\mathrm{t}, 3 \mathrm{H}, J=7.5 \mathrm{~Hz}) ;{ }^{13} \mathrm{C} \mathrm{NMR}\left(100 \mathrm{MHz}, \mathrm{CDCl}_{3}\right) \delta$ 146.1 137.5, 128.4, 128.1, 127.8, 107.8, 89.0, 83.2, 71.4, 57.9, 23.7, 13.3; HRMS (EI) calcd for $\mathrm{C}_{14} \mathrm{H}_{16} \mathrm{O}\left[\mathrm{M}^{+}\right]$200.1201, found 200.1203.

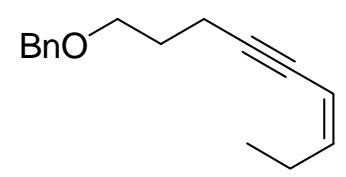

[(Z)-6-Nonen-4-ynyl] benzyl ether (2): ${ }^{1} \mathrm{H}$ NMR (400 MHz, $\left.\mathrm{CDCl}_{3}\right) \delta$ 7.31-7.33 (m, 4H), 7.24-7.27 (m, 1H), $5.80(\mathrm{dt}, 1 \mathrm{H}, J=10.7,7.2 \mathrm{~Hz}), 5.38(\mathrm{dt}, 1 \mathrm{H}, J=10.6,1.4 \mathrm{~Hz}), 4.51(\mathrm{~s}, 2 \mathrm{H})$, $3.58(\mathrm{t}, 2 \mathrm{H}, J=6.2 \mathrm{~Hz}), 2.46(\mathrm{td}, 2 \mathrm{H}, J=7.0,2.1 \mathrm{~Hz}), 2.26(\mathrm{qd}, 2 \mathrm{H}, J=7.5,1.3 \mathrm{~Hz}), 1.80-$ 
$1.87(\mathrm{~m}, 2 \mathrm{H}), 0.98(\mathrm{t}, 3 \mathrm{H}, J=7.5 \mathrm{~Hz}) ;{ }^{13} \mathrm{C} \mathrm{NMR}\left(100 \mathrm{MHz}, \mathrm{CDCl}_{3}\right) \delta 144.3,138.5,128.4$ 127.6, 127.5, 108.6, 93.6, 77.2, 73.0, 68.9, 29.1, 23.4, 16.4, 13.4; HRMS (EI) calcd for $\mathrm{C}_{16} \mathrm{H}_{20} \mathrm{O}\left[\mathrm{M}^{+}\right] 228.1514$, found 228.1518.

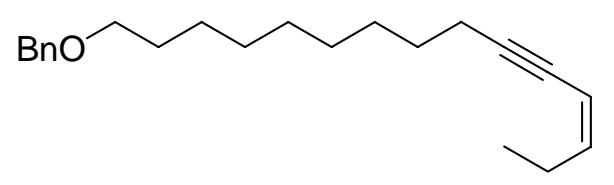

[(Z)-12-pentadecen-10-ynyl] benzyl ether (3): ${ }^{1} \mathrm{H}$ NMR $\left(300 \mathrm{MHz}, \mathrm{CDCl}_{3}\right) \delta$ 7.24-7.34 (m, 5H), $5.79(\mathrm{dt}, 1 \mathrm{H}, J=10.7,7.2 \mathrm{~Hz}), 5.40(\mathrm{~d}, 1 \mathrm{H} J=10.9 \mathrm{~Hz}), 4.49(\mathrm{~s}, 2 \mathrm{H}), 3.46(\mathrm{t}, 2 \mathrm{H}, J=$ $6.6 \mathrm{~Hz}), 2.30(\mathrm{~m}, 4 \mathrm{H}),, 1.51-1.63(\mathrm{~m}, 4 \mathrm{H}), 1.02-1.40(\mathrm{~m}, 10 \mathrm{H}), 0.99(\mathrm{t}, 3 \mathrm{H}, J=7.7 \mathrm{~Hz}) ;{ }^{13} \mathrm{C}$ NMR $\left(75 \mathrm{MHz}, \mathrm{CDCl}_{3}\right) \delta 143.9,138.7,128.3,127.5,127.4,108.7,94.5,77.2,72.8,70.5$, 29.7, 29.4, 29.3, 29.0, 28.9, 28.8, 26.1, 23.4, 19.5, 13.4; HRMS (EI) calcd for $\mathrm{C}_{22} \mathrm{H}_{32} \mathrm{O}\left[\mathrm{M}^{+}\right]$ 312.2453, found 312.2446.

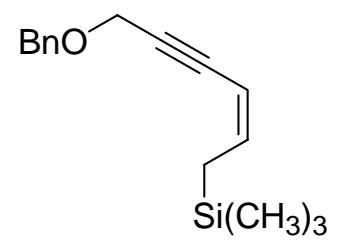

[(Z)-6-Trimethylsilyl-4-hexen-2-ynyl] benzyl ether (4): ${ }^{1} \mathrm{H}$ NMR $\left(300 \mathrm{MHz}, \mathrm{CDCl}_{3}\right) \delta$ 7.19-7.30 (m, 5H), $5.95(\mathrm{dt}, 1 \mathrm{H}, J=10.5,8.9 \mathrm{~Hz}), 5.42(\mathrm{~d}, 1 \mathrm{H}, J=10.9 \mathrm{~Hz}), 4.57(\mathrm{~s}, 2 \mathrm{H})$, $4.28(\mathrm{~d}, 2 \mathrm{H}, J=1.7 \mathrm{~Hz}), 1.81(\mathrm{~d}, 2 \mathrm{H}, J=8.8 \mathrm{~Hz}) 0.01(\mathrm{~s}, 9 \mathrm{H}) ;{ }^{13} \mathrm{C} \mathrm{NMR}\left(100 \mathrm{MHz}, \mathrm{CDCl}_{3}\right) \delta$ $141.8,137.6,128.4,128.1,127.8,105.6,88.8,84.1,71.2,58.0,22.9,-1.6$; HRMS (EI) calcd for $\mathrm{C}_{16} \mathrm{H}_{22} \mathrm{OSi}\left[\mathrm{M}^{+}\right]$258.1440, found 258.1435.

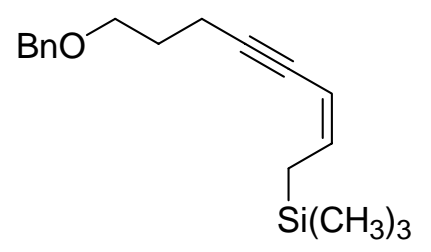


[(Z)-8-Trimethylsilyl-6-octen-4-ynyl] benzyl ether (5): ${ }^{1} \mathrm{H} \mathrm{NMR}\left(300 \mathrm{MHz}, \mathrm{CDCl}_{3}\right) \delta 7.20$ $7.32(\mathrm{~m}, 5 \mathrm{H}), 5.82(\mathrm{dt}, 1 \mathrm{H}, J=10.4,8.8 \mathrm{~Hz}), 5.27(\mathrm{~d}, 1 \mathrm{H}, J=10.5 \mathrm{~Hz}), 4.47(\mathrm{~s}, 2 \mathrm{H}), 3.55(\mathrm{t}$, $2 \mathrm{H}, J=6.3 \mathrm{~Hz}), 2.43(\mathrm{qd}, 2 \mathrm{H}, J=7.0,1.9 \mathrm{~Hz}), 1.76-1.83(\mathrm{~m}, 4 \mathrm{H}), 0.00(\mathrm{~s}, 9 \mathrm{H}) ;{ }^{13} \mathrm{C} \mathrm{NMR}$ $\left(100 \mathrm{MHz}, \mathrm{CDCl}_{3}\right) \delta 139.5,138.5,128.3,127.7,127.6,106.5,93.2,78.2,73.0,69.0,29.2$, 22.4, 16.4, -1.6; HRMS (EI) calcd for $\mathrm{C}_{18} \mathrm{H}_{26} \mathrm{OSi}\left[\mathrm{M}^{+}\right]$286.1753, found 286.1751.

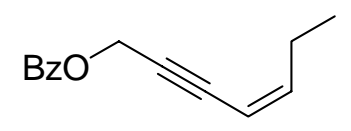

[(Z)-4-Hepten-2-ynyl] benzoate (6): ${ }^{1} \mathrm{H}$ NMR $\left(400 \mathrm{MHz}, \mathrm{CDCl}_{3}\right) \delta 8.07$ (d, 2H, $\left.J=7.7 \mathrm{~Hz}\right)$, $7.55(\mathrm{t}, 1 \mathrm{H}, J=7.5 \mathrm{~Hz}), 7.43(\mathrm{t}, 2 \mathrm{H}, J=7.5 \mathrm{~Hz}), 5.95(\mathrm{dt}, 1 \mathrm{H}, J=10.8,7.4 \mathrm{~Hz}), 5.45(\mathrm{dt}, 1 \mathrm{H}$, $J=10.8,1.8 \mathrm{~Hz}), 5.06(\mathrm{~d}, 2 \mathrm{H}, J=2.1 \mathrm{~Hz}), 2.31(\mathrm{qd}, 2 \mathrm{H}, J=6.9,1.3 \mathrm{~Hz}), 1.00(\mathrm{t}, 3 \mathrm{H}, J=7.6$ $\mathrm{Hz}) ;{ }^{13} \mathrm{C}$ NMR $\left(100 \mathrm{MHz}, \mathrm{CDCl}_{3}\right) \delta 165.9,147.1,133.1,129.7,129.6,128.3,107.5,86.9$, 83.4, 53.4, 23.7, 13.2; HRMS (EI) calcd for $\mathrm{C}_{14} \mathrm{H}_{14} \mathrm{O}_{2}\left[\mathrm{M}^{+}\right]$214.0994, found 214.0995.

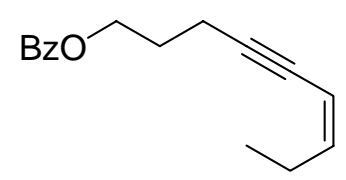

[(Z)-6-Nonen-4-ynyl] benzoate (7): ${ }^{1} \mathrm{H} \mathrm{NMR}\left(300 \mathrm{MHz}, \mathrm{CDCl}_{3}\right) \delta 8.05(\mathrm{~d}, 2 \mathrm{H}, J=7.8 \mathrm{~Hz})$, $7.55(\mathrm{t}, 1 \mathrm{H}, J=7.4 \mathrm{~Hz}), 7.43(\mathrm{t}, 2 \mathrm{H}, J=7.5 \mathrm{~Hz}), 5.83(\mathrm{dt}, 1 \mathrm{H}, J=10.6,7.3 \mathrm{~Hz}), 5.40(\mathrm{~d}, 1 \mathrm{H}, J$ $=10.6 \mathrm{~Hz}), 4.44(\mathrm{t}, 2 \mathrm{H}, J=6.3 \mathrm{~Hz}), 2.39(\mathrm{td}, 2 \mathrm{H}, J=7.00,1.8 \mathrm{~Hz}), 2.24-2.34(\mathrm{qd}, 2 \mathrm{H}, J=7.5$, $0.9 \mathrm{~Hz}), 1.97-2.06(\mathrm{~m}, 2 \mathrm{H}), 1.00(\mathrm{t}, 3 \mathrm{H}, J=7.5 \mathrm{~Hz}) ;{ }^{13} \mathrm{C} \mathrm{NMR}\left(100 \mathrm{MHz}, \mathrm{CDCl}_{3}\right) \delta 166.3$, $144.3,132.7,130.4,129.5,128.2,108.5,92.5,78.0,63.6,28.1,23.3,16.4,13.2$; HRMS (EI) calcd for $\mathrm{C}_{16} \mathrm{H}_{18} \mathrm{O}_{2}\left[\mathrm{M}^{+}\right]$242.1307, found 242.1334.

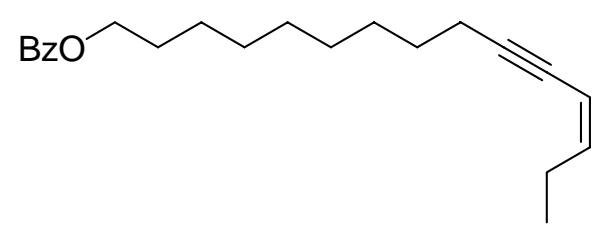


[(Z)-12-Pentadecen-10-ynyl] benzoate (8): ${ }^{1} \mathrm{H}$ NMR $\left(300 \mathrm{MHz}, \mathrm{CDCl}_{3}\right) \delta 8.02(\mathrm{~d}, 2 \mathrm{H}, J=$ $7.9 \mathrm{~Hz}), 7.50(\mathrm{t}, 1 \mathrm{H}, J=7.7 \mathrm{~Hz}), 7.42(\mathrm{t}, 2 \mathrm{H}, J=7.2 \mathrm{~Hz}), 5.77(\mathrm{dt}, 1 \mathrm{H}, J=10.7,7.2 \mathrm{~Hz}), 5.39$ $(\mathrm{d}, 1 \mathrm{H}, J=10.8 \mathrm{~Hz}), 4.29(\mathrm{t}, 2 \mathrm{H}, J=6.6 \mathrm{~Hz}), 2.25-2.31(\mathrm{~m}, 4 \mathrm{H}), 1.76-1.72(\mathrm{~m}, 2 \mathrm{H}), 1.30-1.51$ (m, 12H), $0.97(\mathrm{t}, 3 \mathrm{H}, J=7.8 \mathrm{~Hz}) ;{ }^{13} \mathrm{C} \mathrm{NMR}\left(75 \mathrm{MHz}, \mathrm{CDCl}_{3}\right) \delta 166.6,144.0,132.7,130.5$, $129.5,128.2,108.7,94.4,77.2,65.0,29.3,29.2,29.0,28.8,28.8,28.7,26.0,23.4,19.5,13.4$; HRMS (EI) calcd for $\mathrm{C}_{22} \mathrm{H}_{30} \mathrm{O}_{2}\left[\mathrm{M}^{+}\right]$326.2246, found 326.2248.

$\mathrm{BzO}^{\mathrm{Si}\left(\mathrm{CH}_{3}\right)_{3}}$

[(Z)-6-Trimethylsilyl-4-hexen-2-ynyl] benzoate (9): ${ }^{1} \mathrm{H} \mathrm{NMR}\left(300 \mathrm{MHz}, \mathrm{CDCl}_{3}\right) \delta 8.04$ (d, $2 \mathrm{H}, J=7.2 \mathrm{~Hz}), 7.53(\mathrm{t}, 1 \mathrm{H}, J=7.4 \mathrm{~Hz}), 7.40(\mathrm{t}, 2 \mathrm{H}, J=7.8 \mathrm{~Hz}), 6.01(\mathrm{dt}, 1 \mathrm{H}, J=10.5,8.8$ $\mathrm{Hz}), 5.34(\mathrm{~d}, 1 \mathrm{H}, J=10.6 \mathrm{~Hz}), 5.04(\mathrm{~d}, 2 \mathrm{H}, J=1.8 \mathrm{~Hz}), 1.82(\mathrm{~d}, 2 \mathrm{H}, J=8.8 \mathrm{~Hz}), 0.00(\mathrm{~s}, 9 \mathrm{H})$; ${ }^{13} \mathrm{C}$ NMR $\left(100 \mathrm{MHz}, \mathrm{CDCl}_{3}\right) \delta 165.9,143.1,133.1,129.8,129.7,128.3,105.1,86.7,84.4$, 53.6, 23.0, -1.7; HRMS (EI) calcd for $\mathrm{C}_{16} \mathrm{H}_{20} \mathrm{O}_{2} \mathrm{Si}\left[\mathrm{M}^{+}\right]$272.1233, found 272.1222.

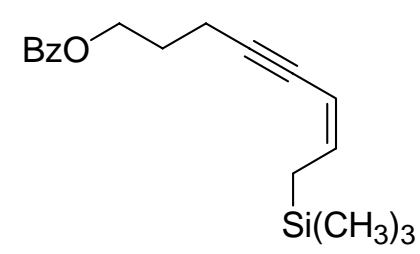

[(Z)-8-Trimethylsilyl-6-octen-4-ynyl] benzoate (10): ${ }^{1} \mathrm{H}$ NMR $\left(300 \mathrm{MHz}, \mathrm{CDCl}_{3}\right) \delta 8.00(\mathrm{~d}$, $2 \mathrm{H}, J=7.1 \mathrm{~Hz}), 7.51(\mathrm{t}, 1 \mathrm{H}, J=7.5 \mathrm{~Hz}), 7.39(\mathrm{t}, 2 \mathrm{H}, J=7.6 \mathrm{~Hz}), 5.85(\mathrm{dt}, 1 \mathrm{H}, J=10.4,8.7$ $\mathrm{Hz}), 5.28(\mathrm{~d}, 1 \mathrm{H}, J=10.6 \mathrm{~Hz}), 4.41(\mathrm{t}, 2 \mathrm{H}, J=6.3 \mathrm{~Hz}), 2.51(\mathrm{qd}, 2 \mathrm{H}, J=7.0,1.8 \mathrm{~Hz}), 1.92-$ $2.02(\mathrm{~m}, 2 \mathrm{H}), 1.77(\mathrm{~d}, 2 \mathrm{H}, J=8.7 \mathrm{~Hz}), 0.00(\mathrm{~s}, 9 \mathrm{H}) ;{ }^{13} \mathrm{C} \mathrm{NMR}\left(100 \mathrm{MHz}, \mathrm{CDCl}_{3}\right) \delta 166.4$, $139.9,132.8,130.2,129.5,128.3,106.2,92.2,78.8,63.7,28.2,22.5,16.4,-1.7$; HRMS (EI) calcd for $\mathrm{C}_{18} \mathrm{H}_{24} \mathrm{O}_{2} \mathrm{Si}\left[\mathrm{M}^{+}\right]$300.1546, found 300.1550 . 
<smiles>C/C=C\C#CCN(CC)C(=O)c1ccccc1</smiles>

$\boldsymbol{N}$-Ethyl- $\boldsymbol{N}$-[(Z)-4-hepten-2-ynyl] benzamide (11): ${ }^{1} \mathrm{H}$ NMR $\left(300 \mathrm{MHz}, \mathrm{CDCl}_{3}\right) \delta$ 7.36-7.48 (m, 5H), $5.95(\mathrm{dt}, 1 \mathrm{H}, J=10.2,7.4 \mathrm{~Hz}), 5.44(\mathrm{~d}, 1 \mathrm{H}, 10.7 \mathrm{~Hz}), 4.33(\mathrm{~b}, 2 \mathrm{H}), 3.57$ (b, 2H), $2.32(\mathrm{qd}, 2 \mathrm{H}, J=7.5,1.1 \mathrm{~Hz}), 1.24(\mathrm{~b}, 3 \mathrm{H}), 1.03(\mathrm{t}, 3 \mathrm{H}, J=7.6 \mathrm{~Hz}) ;{ }^{13} \mathrm{C}$ NMR $(100 \mathrm{MHz}$, $\left.\mathrm{CDCl}_{3}\right) \delta 170.8,146.4,136.1,129.6,128.3,107.7,88.2,77.2,42.9,40.2,33.9,23.6,13.3$, 12.4; HRMS (EI) calcd for $\mathrm{C}_{16} \mathrm{H}_{19} \mathrm{NO}\left[\mathrm{M}^{+}\right]$241.1467, found 241.1460.

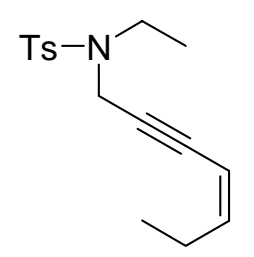

$\boldsymbol{N}$-Ethyl- $\boldsymbol{N}$-[(Z)-4-hepten-2-ynyl] $\boldsymbol{p}$-toluenesulfonamide (12): ${ }^{1} \mathrm{H} \mathrm{NMR}\left(300 \mathrm{MHz}, \mathrm{CDCl}_{3}\right)$ $\delta 7.73(\mathrm{~d}, 2 \mathrm{H}, J=8.3 \mathrm{~Hz}), 7.27(\mathrm{~d}, 2 \mathrm{H}, J=8.0 \mathrm{~Hz}), 5.81(\mathrm{dt}, 1 \mathrm{H}, J=10.7,7.4 \mathrm{~Hz}), 5.18(\mathrm{~d}$, $1 \mathrm{H}, J=10.7 \mathrm{~Hz}), 4.30(\mathrm{~d}, 2 \mathrm{H}, J=1.7 \mathrm{~Hz}), 3.28(\mathrm{q}, 2 \mathrm{H}, J=7.2 \mathrm{~Hz}), 2.40(\mathrm{~s}, 3 \mathrm{H}), 2.03(\mathrm{qd}, 2 \mathrm{H}$, $J=7.5,1.1 \mathrm{~Hz}), 1.20(\mathrm{t}, 3 \mathrm{H}, J=7.2 \mathrm{~Hz}), 0.92(\mathrm{t}, 3 \mathrm{H}, J=7.5 \mathrm{~Hz}) ;{ }^{13} \mathrm{C} \mathrm{NMR}(100 \mathrm{MHz}$, $\left.\mathrm{CDCl}_{3}\right) \delta 145.8,143.1,135.9,129.3,127.5,107.3,85.9,82.0,41.1,36.5,23.4,21.4,13.2$, 13.1; HRMS (EI) calcd for $\mathrm{C}_{16} \mathrm{H}_{21} \mathrm{NO}_{2} \mathrm{~S}\left[\mathrm{M}^{+}\right] 291.1293$, found 291.1312.

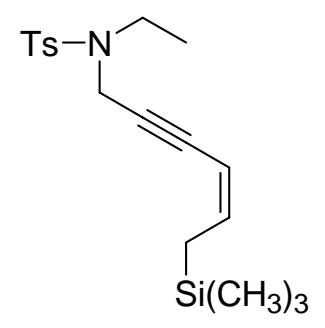

$N$-Ethyl- $N$-[6-trimethylsilyl-(Z)-4-hexen-2-ynyl] $p$-toluenesulfonamide (13): ${ }^{1} \mathrm{H}$ NMR $\left(300 \mathrm{MHz}, \mathrm{CDCl}_{3}\right) \delta 7.74(\mathrm{~d}, 2 \mathrm{H}, J=8.2 \mathrm{~Hz}), 7.28(\mathrm{~d}, 2 \mathrm{H}, J=7.8 \mathrm{~Hz}), 5.89(\mathrm{dt}, 1 \mathrm{H}, J=10.4$, 
$9.2 \mathrm{~Hz}), 5.10(\mathrm{~d}, 1 \mathrm{H}, J=10.7 \mathrm{~Hz}), 4.32(\mathrm{~d}, 2 \mathrm{H}, J=1.3 \mathrm{~Hz}), 3.29(\mathrm{q}, 2 \mathrm{H}, J=7.1 \mathrm{~Hz}), 2.41(\mathrm{~s}$, $3 \mathrm{H}), 1.62(\mathrm{~d}, 2 \mathrm{H}, J=8.7 \mathrm{~Hz}), 1.20(\mathrm{t}, 3 \mathrm{H}, J=7.1 \mathrm{~Hz}), 0.02(\mathrm{~s}, 9 \mathrm{H}) ;{ }^{13} \mathrm{C} \mathrm{NMR}(100 \mathrm{MHz}$, $\left.\mathrm{CDCl}_{3}\right) \delta 143.1,141.5,136.0,129.4,127.7,105.1,85.5,82.9,41.1,36.6,22.7,21.4,13.1,-$ 1.8; HRMS (EI) calcd for $\mathrm{C}_{18} \mathrm{H}_{27} \mathrm{NO}_{2} \mathrm{SSi}\left[\mathrm{M}^{+}\right]$349.1532, found 349.1531.

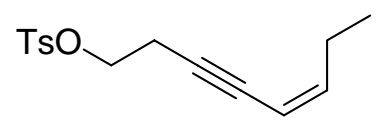

[(Z)-5-octen-3-ynyl] tosylate (15) ${ }^{1} \mathrm{H}$ NMR $\left(300 \mathrm{MHz}, \mathrm{CDCl}_{3}\right) 7.79$ (d, 2H, J=8.2 Hz), 7.33 $(\mathrm{d}, 2 \mathrm{H}, J=8.2 \mathrm{~Hz}), 5.84(\mathrm{dt}, 1 \mathrm{H}, J=10.7,7.4 \mathrm{~Hz}), 5.31(\mathrm{~d}, 1 \mathrm{H}, J=10.7 \mathrm{~Hz}), 4.10(\mathrm{t}, 2 \mathrm{H}, J=$ $7.2 \mathrm{~Hz}), 2.69(\mathrm{td}, 2 \mathrm{H}, J=7.1,1.9 \mathrm{~Hz}), 2.43(\mathrm{~s}, 3 \mathrm{H}), 2.22(\mathrm{t}, 2 \mathrm{H}, J=7.4 \mathrm{~Hz}), 0.97(\mathrm{t}, 3 \mathrm{H}, J=$ $7.5 \mathrm{~Hz}) ;{ }^{13} \mathrm{C} \mathrm{NMR}\left(100 \mathrm{MHz}, \mathrm{CDCl}_{3}\right)$ 145.4, 144.8, 132.8, 129.8, 127.8, 107.8, 87.8, 79.9, 67.7, 23.4, 21.4, 20.3, 13.2; HRMS (EI) calcd for $\mathrm{C}_{15} \mathrm{H}_{18} \mathrm{O}_{3} \mathrm{~S}\left[\mathrm{M}^{+}\right]$278.0977, found 278.0970. 
Table S-1. Calculated energies (in hartree) of chelation and non-chelation metallacyclobutane intermediates of the reaction derived from Scheme 3 in the text. ${ }^{a}$

\begin{tabular}{|c|c|c|c||c|c|c|}
\hline \multirow{2}{*}{ method } & \multicolumn{3}{|c||}{$(\mathrm{n}=1)$ substrate } & \multicolumn{3}{c|}{$(\mathrm{n}=3)$ substrate } \\
\cline { 2 - 7 } & $\begin{array}{c}\text { chelation } \\
(\mathrm{A})\end{array}$ & $\begin{array}{c}\text { non-chelation } \\
(\mathrm{B})\end{array}$ & $\begin{array}{c}\text { (A)-(B) } \\
\text { chelation } \\
(\mathrm{A})\end{array}$ & $\begin{array}{c}\text { non-chelation } \\
(\mathrm{B})\end{array}$ & $(\mathrm{A})-(\mathrm{B})$ \\
\hline HF & -7522.3206 & -7522.3004 & -0.0202 & -7600.0707 & -7599.9436 & -0.1271 \\
\hline SVWN5 $^{3}$ & -7513.8146 & -7513.8055 & -0.0092 & -7591.2684 & -7591.2475 & -0.0210 \\
\hline B3LYP $^{4}$ & -7538.6523 & -7538.6467 & -0.0056 & -7616.8543 & -7616.8458 & -0.0085 \\
\hline B3P86 & -7546.4100 & -7546.4232 & 0.0132 & -7624.9339 & -7624.9132 & -0.0207 \\
\hline HF* & -7577.3298 & -7577.3180 & -0.0118 & -7635.4842 & -7635.3638 & -0.1204 \\
\hline
\end{tabular}

${ }^{a}$ All energies were calculated at the HF optimized geometry with $3-21 G^{* *}$ basis set. ${ }^{2}$ All except $\mathrm{HF}^{*}$ are calculated with the $3-21 \mathrm{G}^{* *}$ basis set and $\mathrm{HF}^{*}$ refers to calculation with the DGDZVP basis set. 
[(Z)-4-Hepten-2-ynyl] benzyl ether (1)

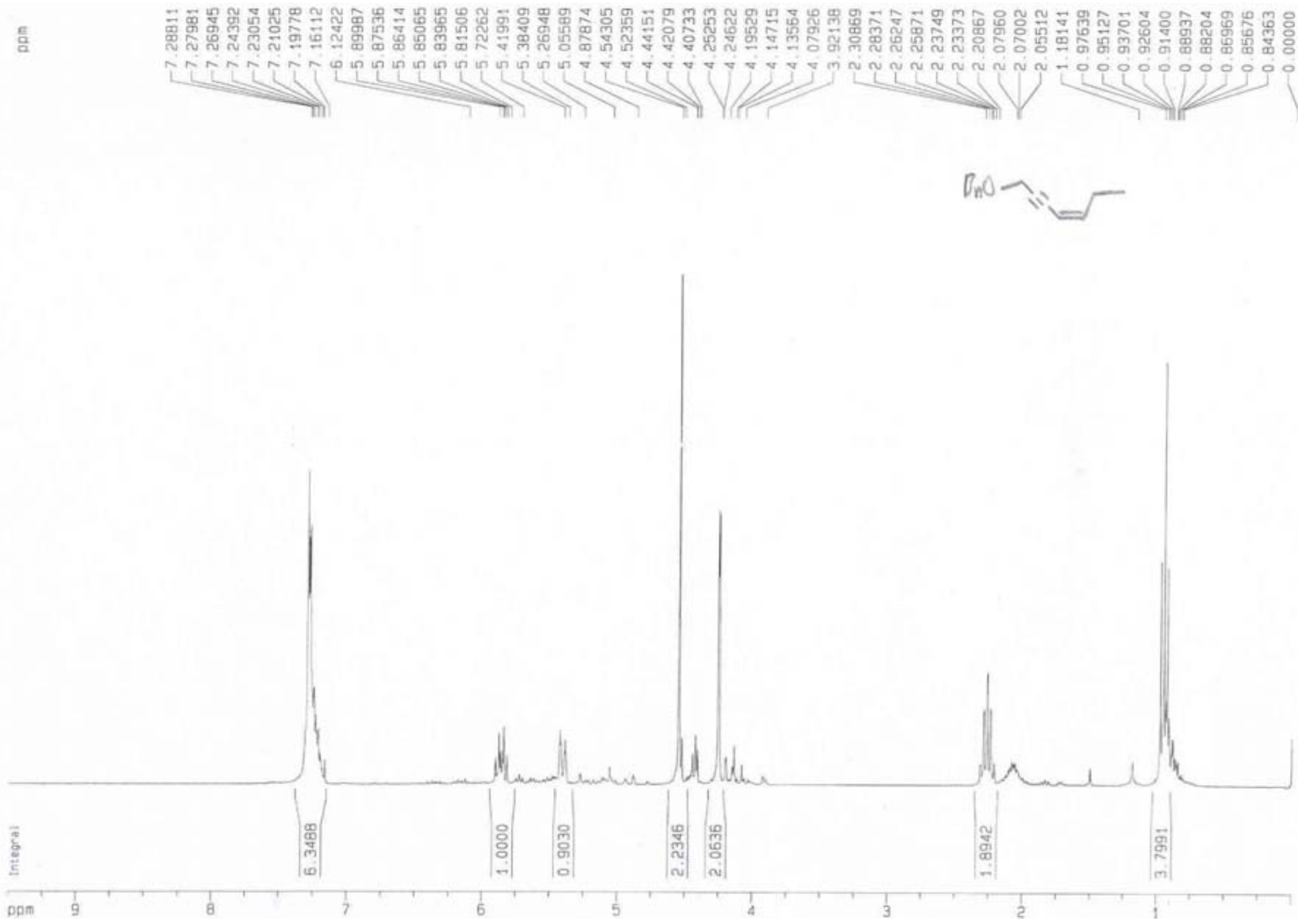

言

$$
?
$$

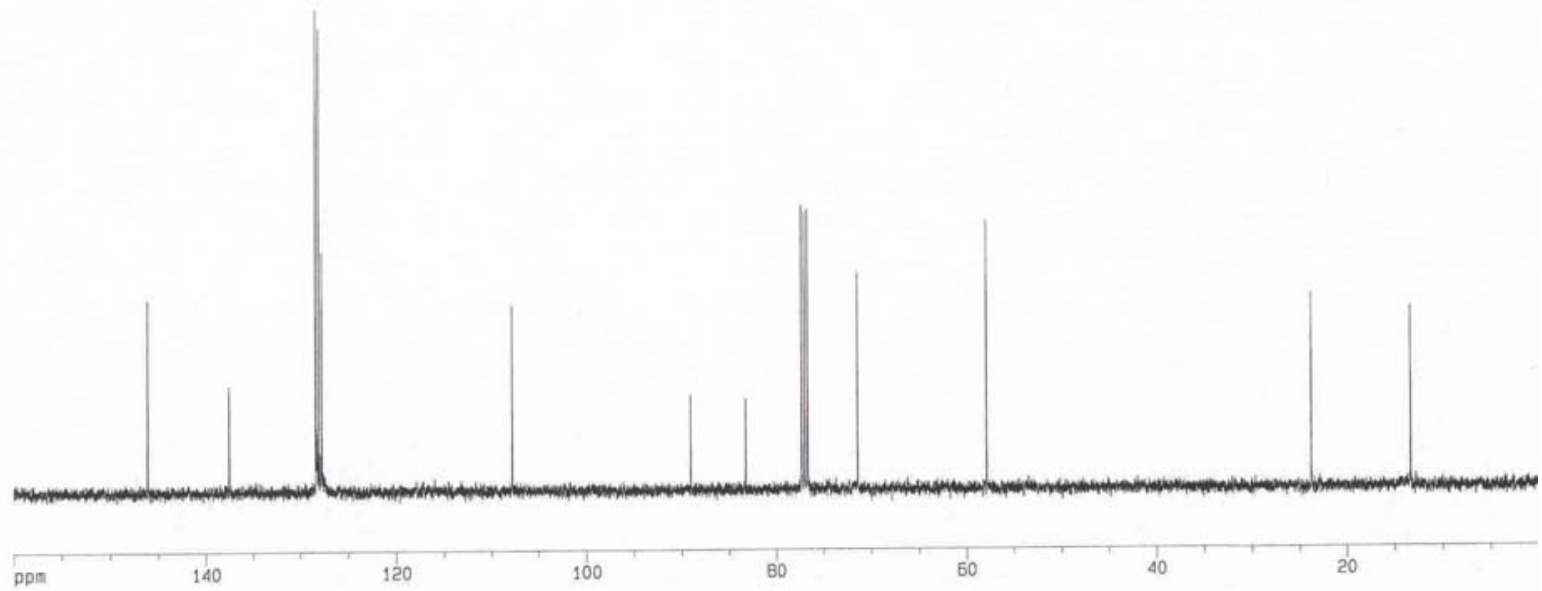


[(Z)-6-Nonen-4-ynyl] benzyl ether (2)

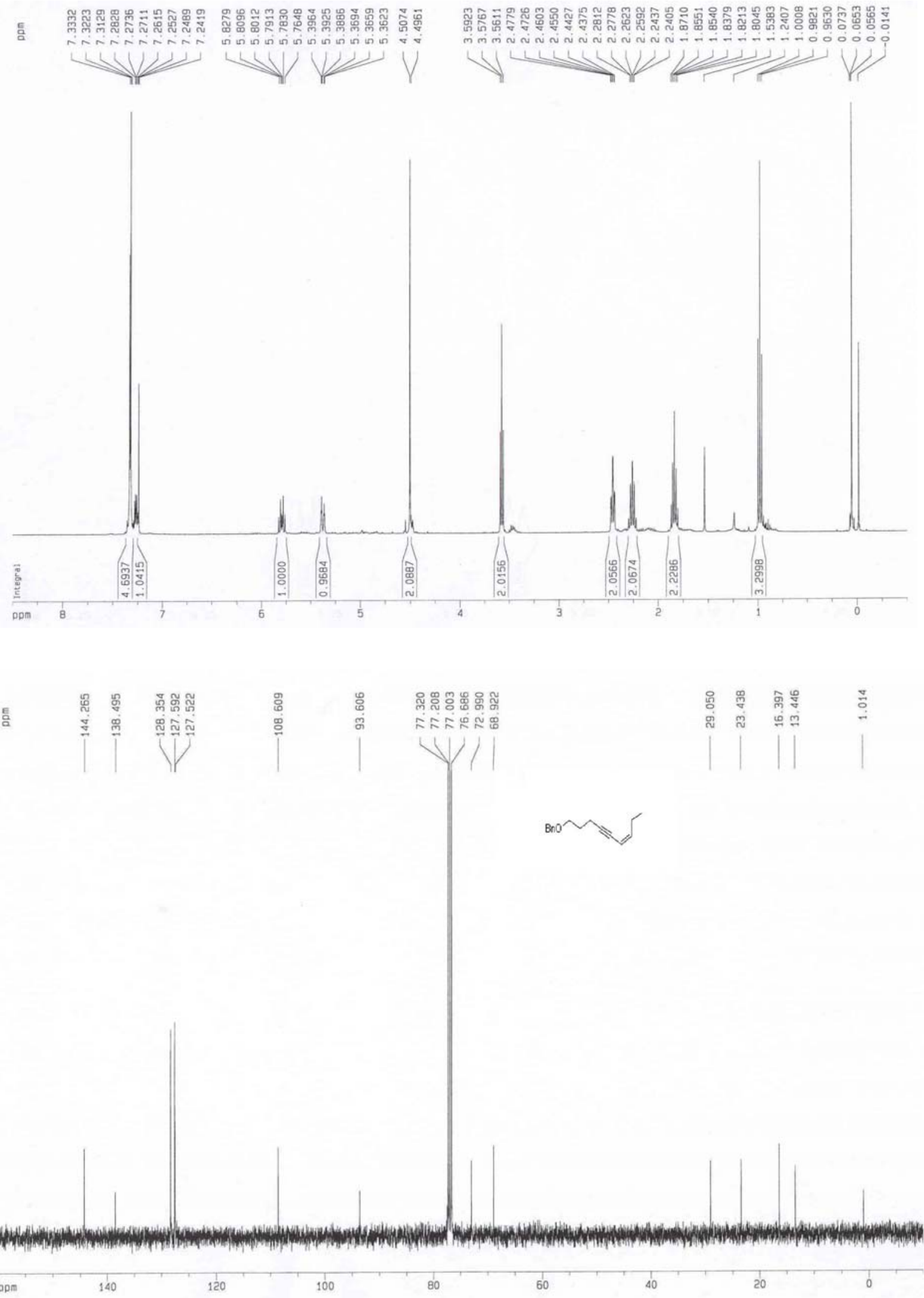


[(Z)-12-pentadecen-10-ynyl] benzyl ether (3)
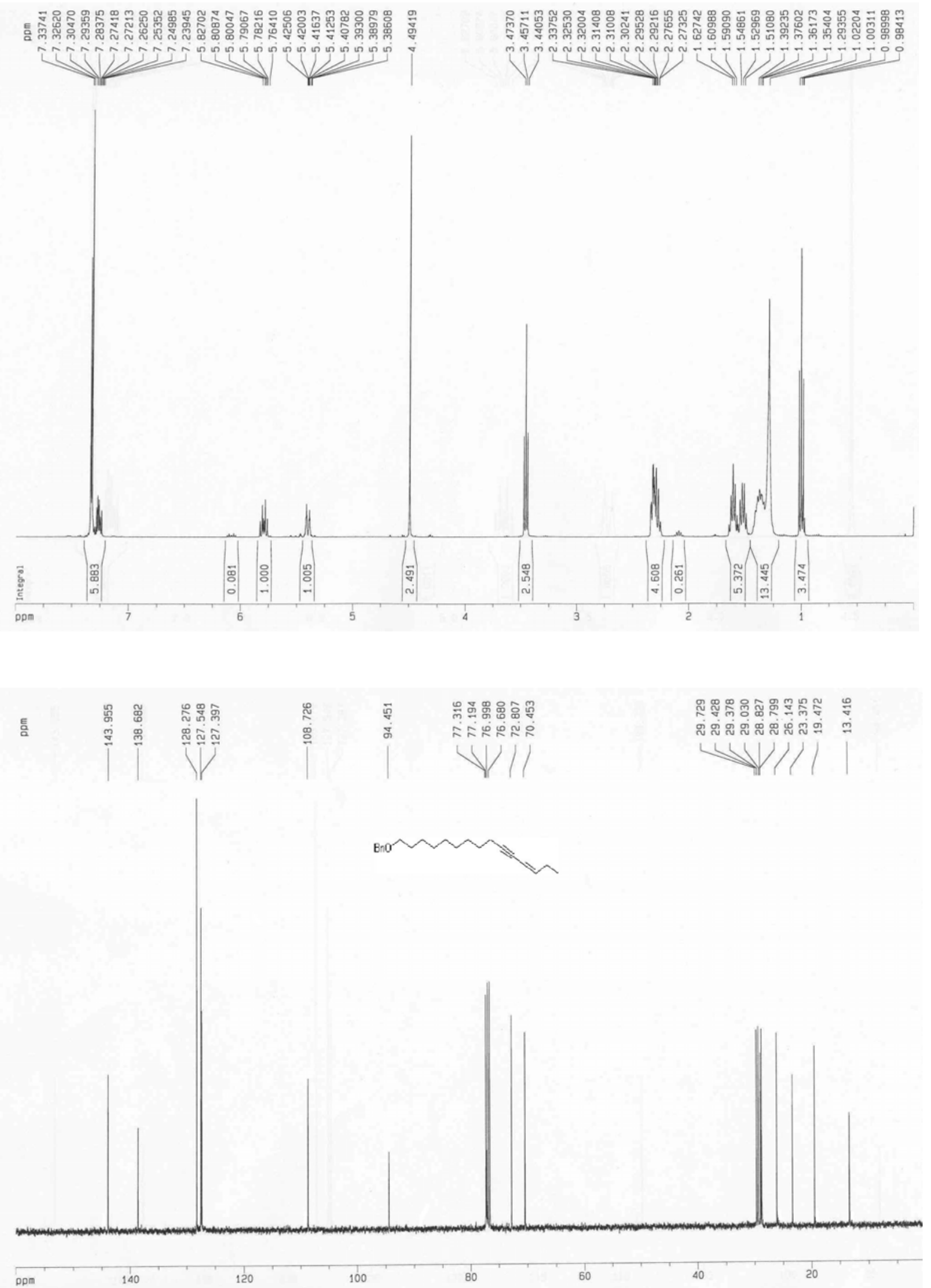
[(Z)-6-Trimethylsilyl-4-hexen-2-ynyl] benzyl ether (4)

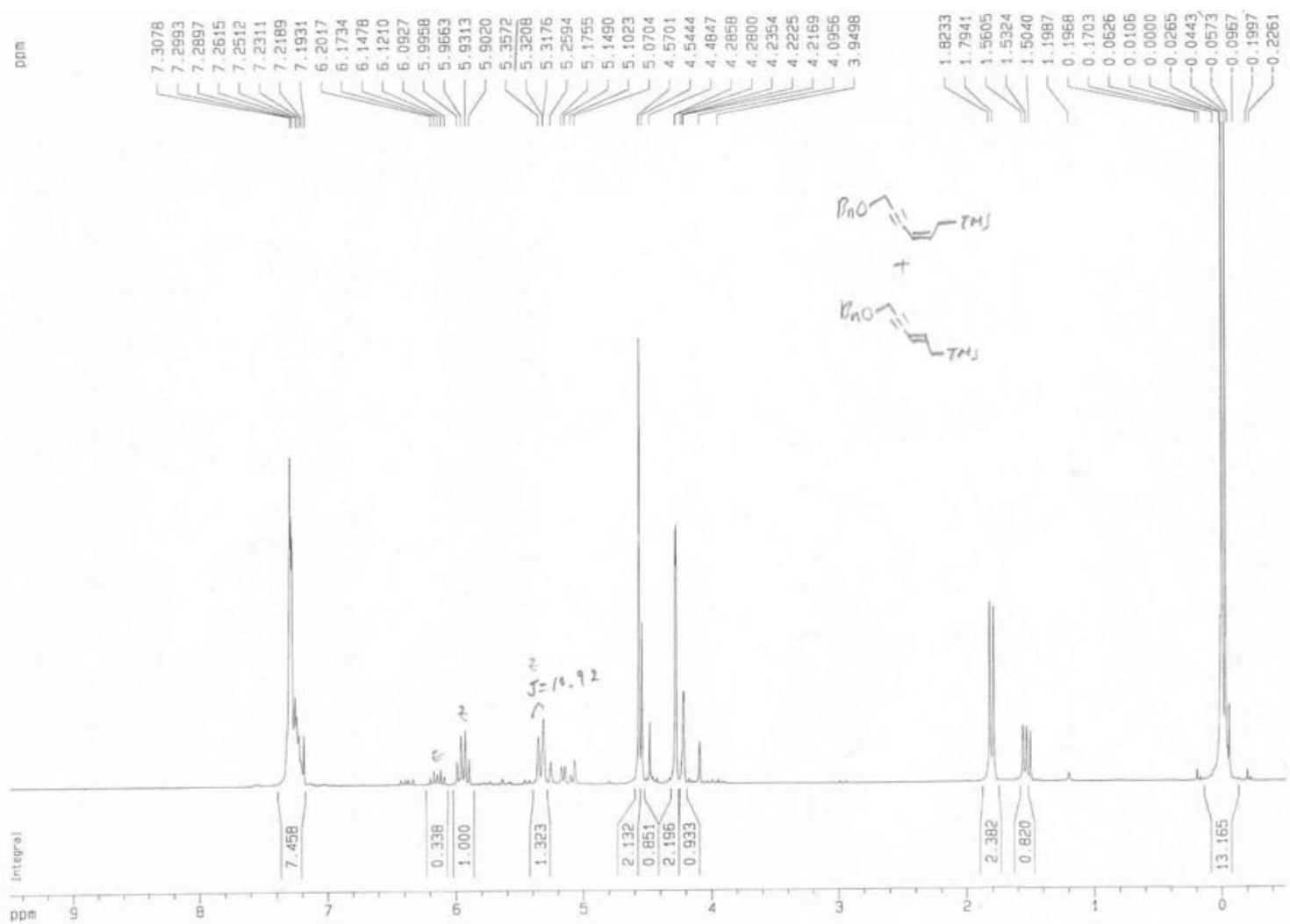

高
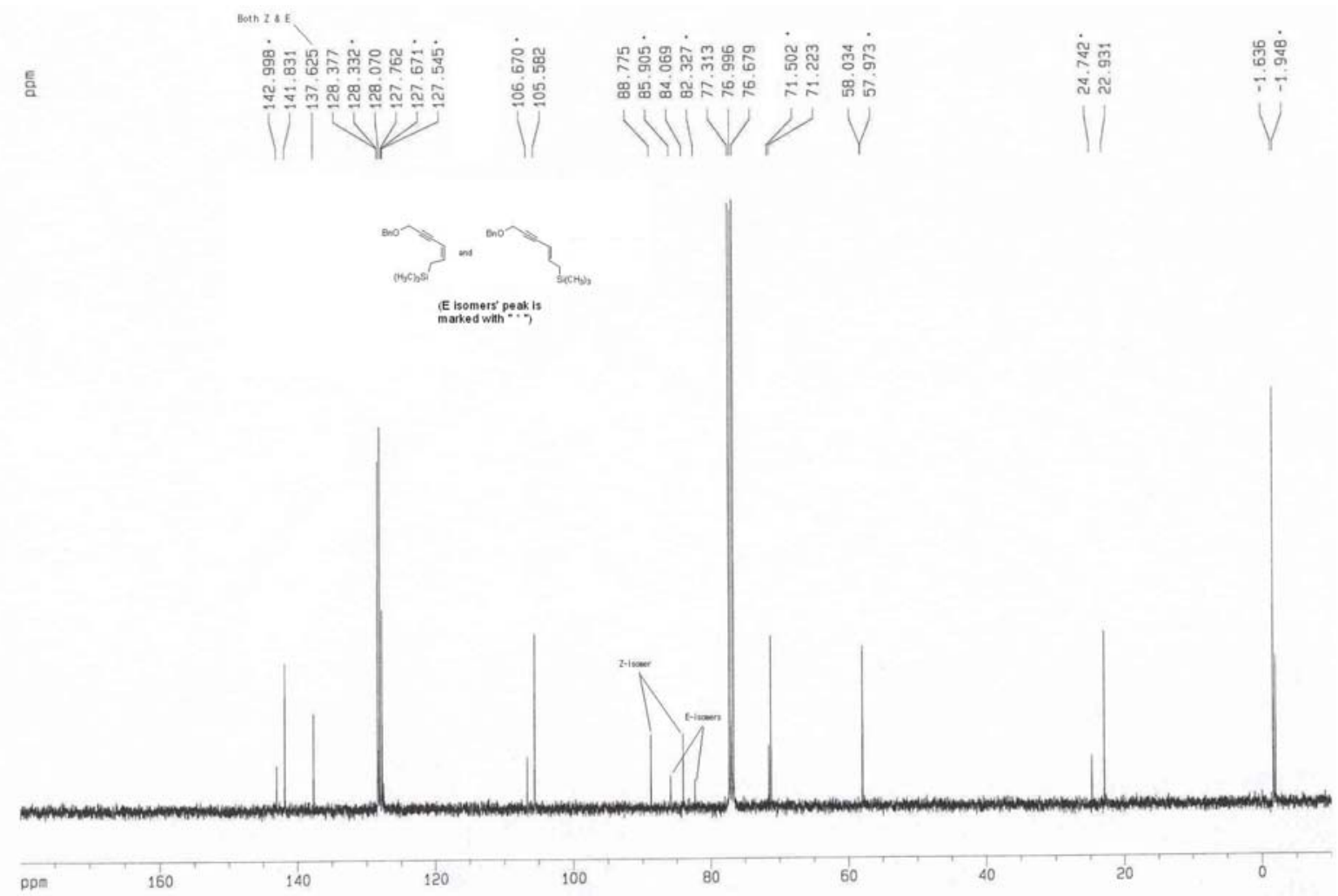
[(Z)-8-Trimethylsilyl-6-octen-4-ynyl] benzyl ether (5)
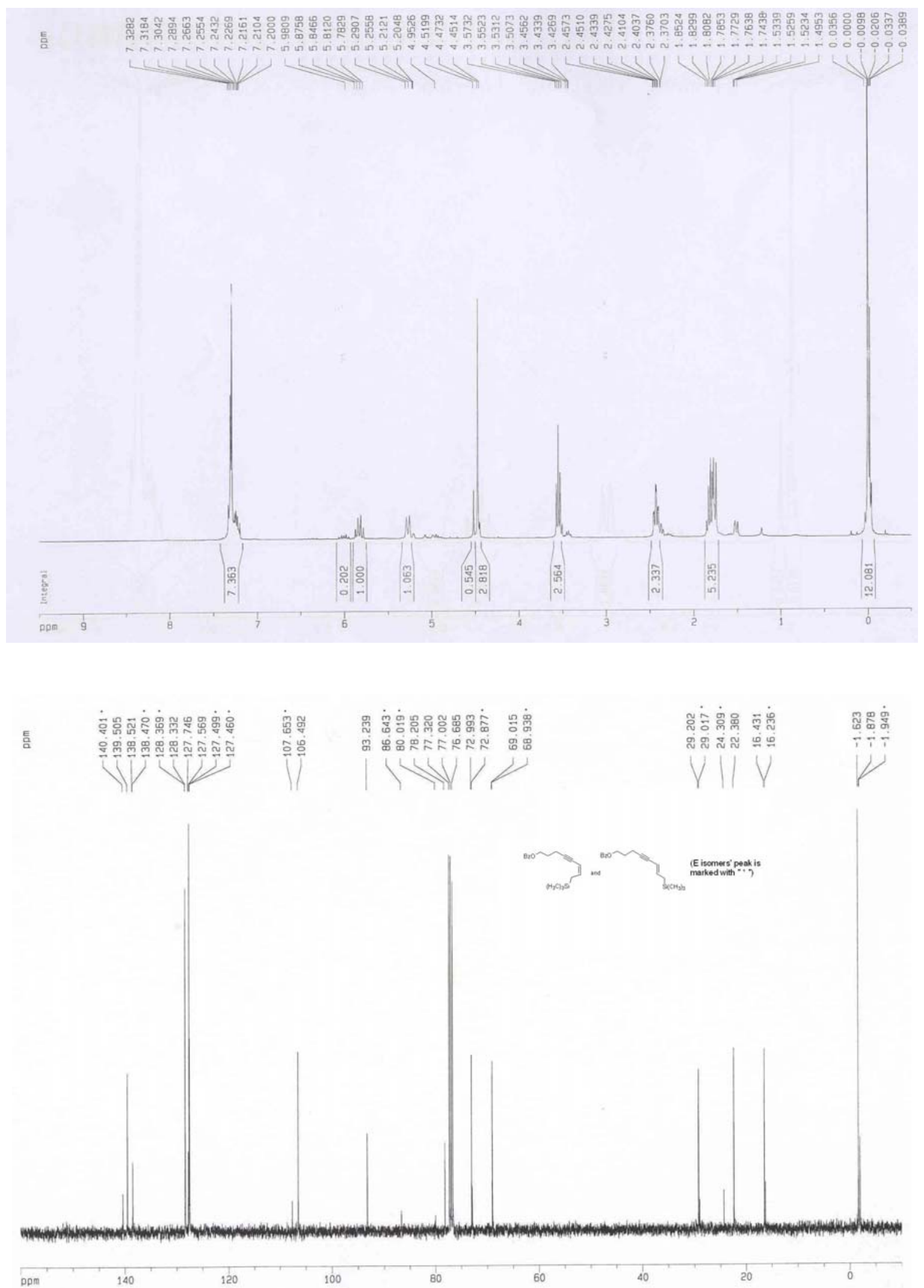
[(Z)-4-Hepten-2-ynyl] benzoate (6)
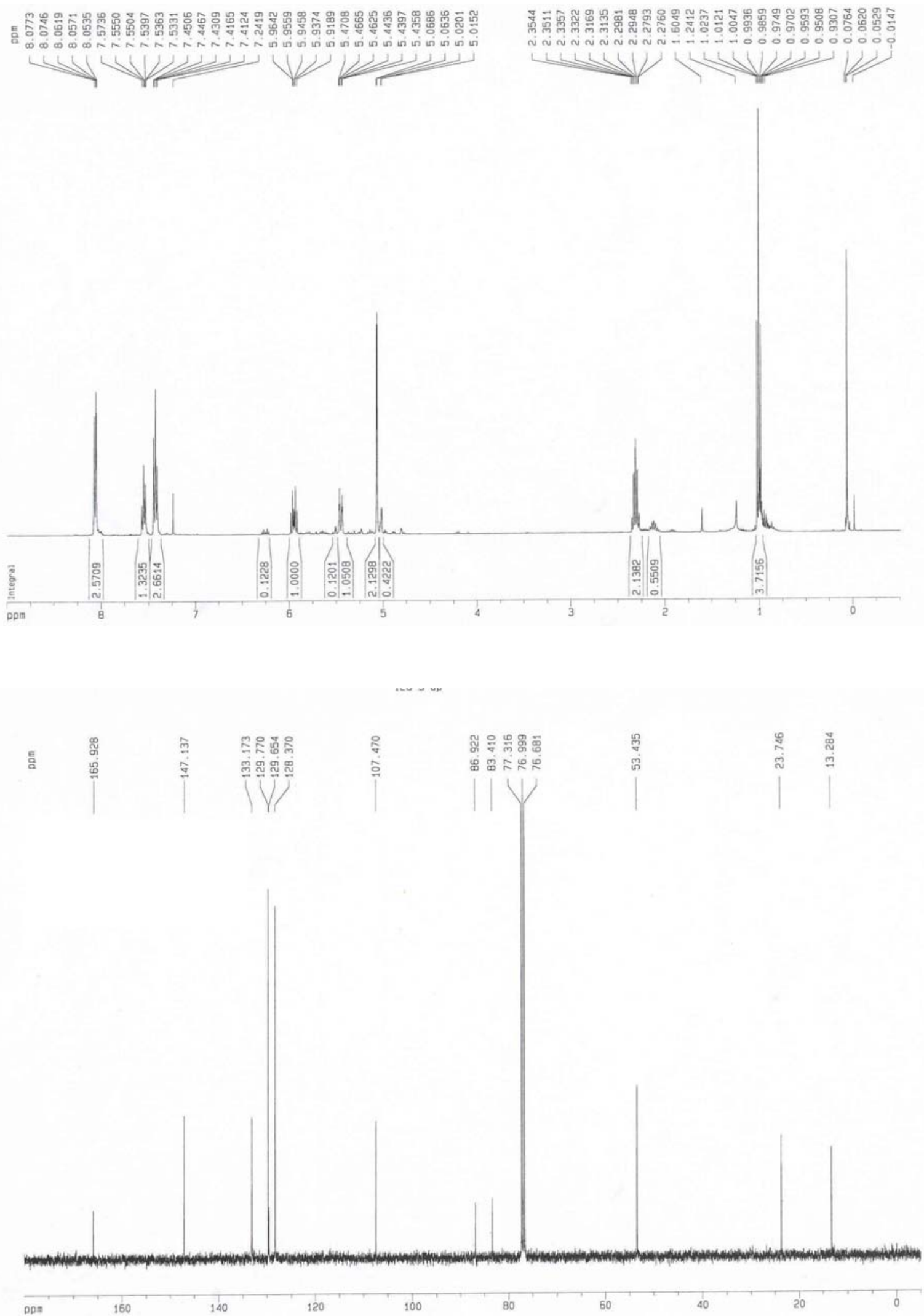
[(Z)-6-Nonen-4-ynyl] benzoate (7)
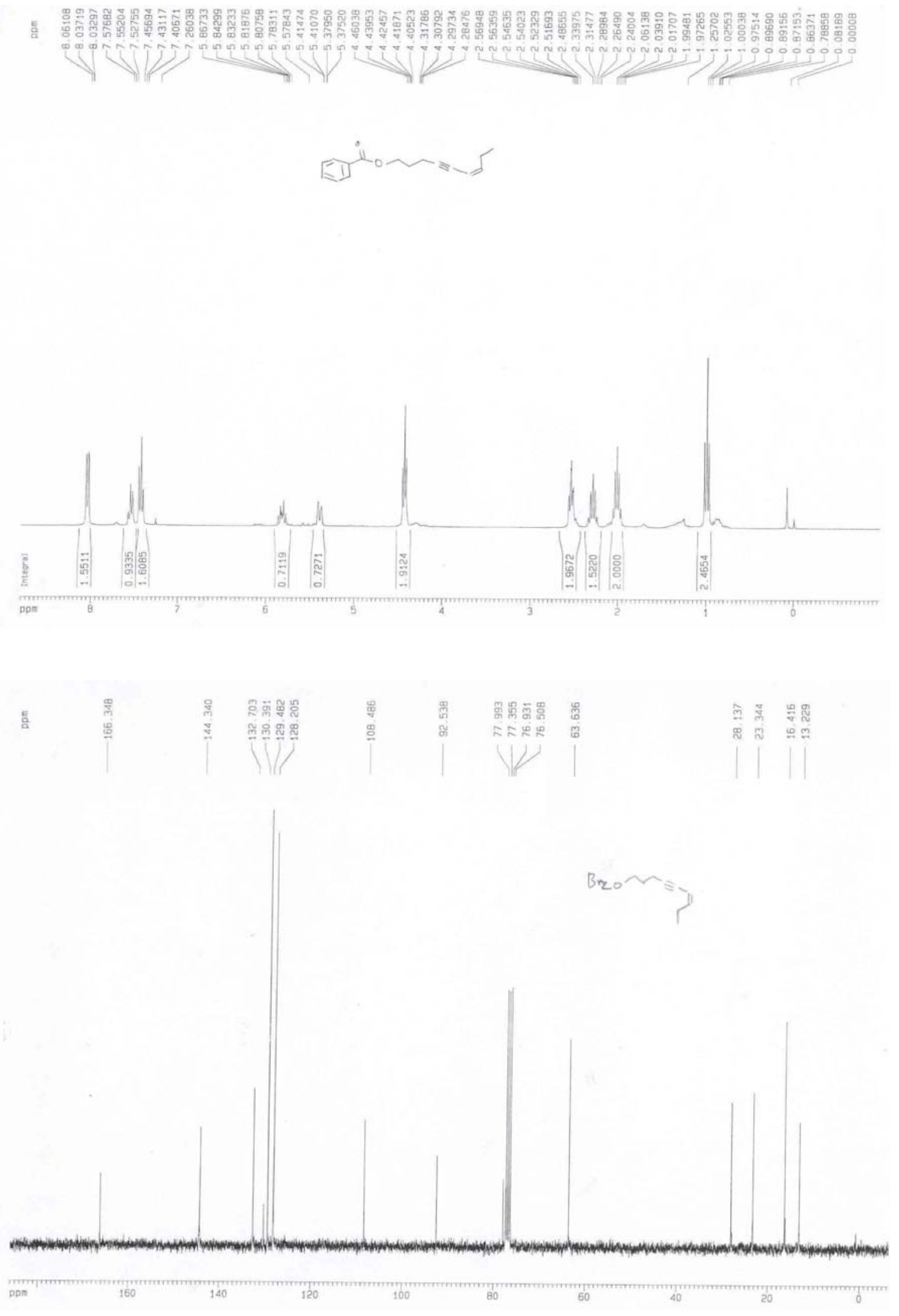
[(Z)-12-Pentadecen-10-ynyl] benzoate (8)

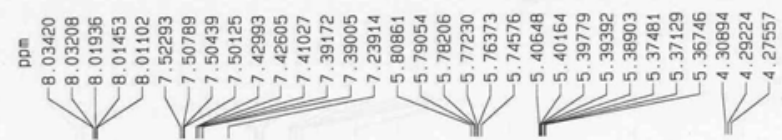

\%

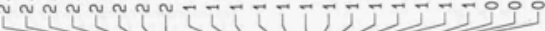
$+1$.

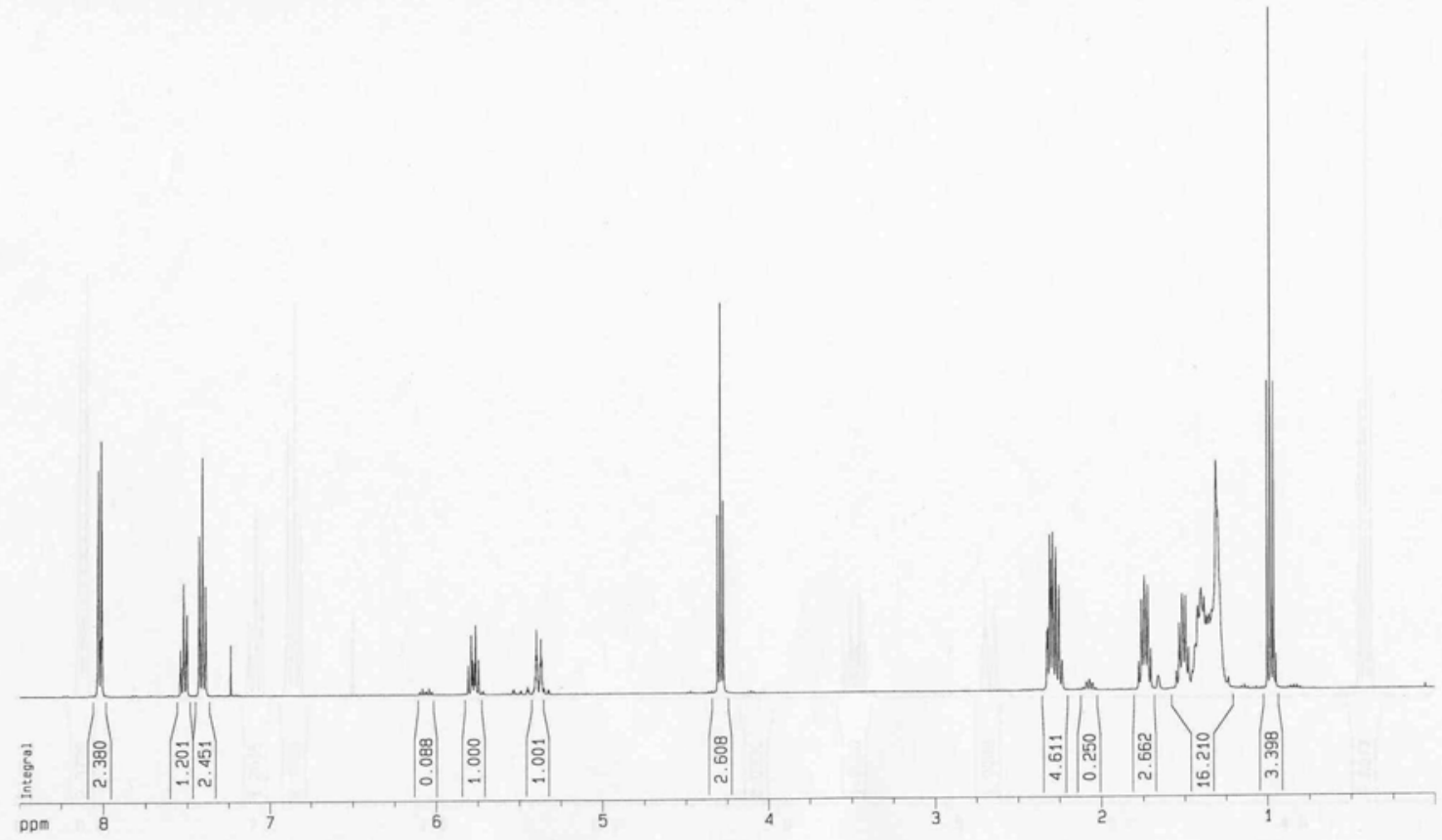

E

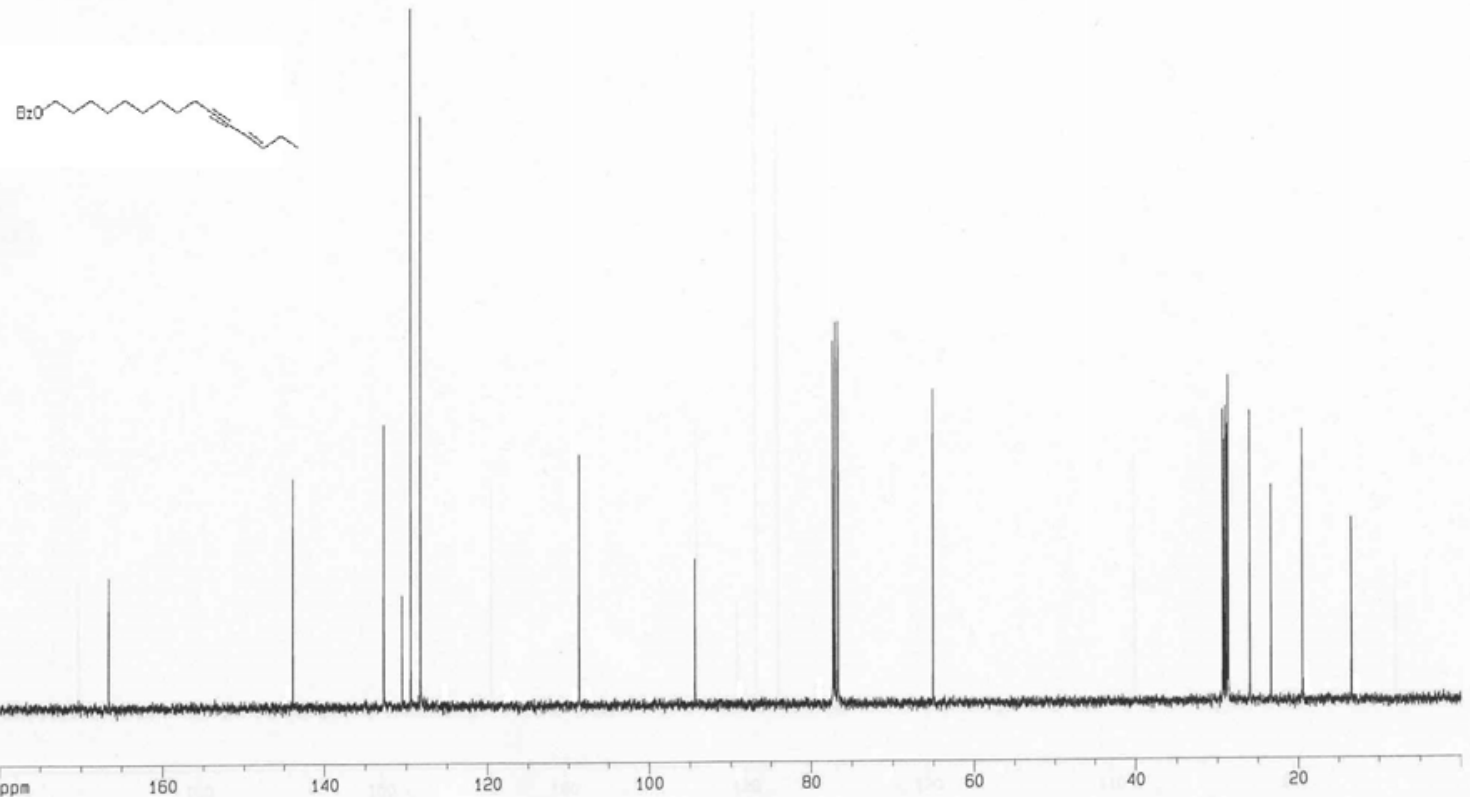


[(Z)-6-Trimethylsilyl-4-hexen-2-ynyl] benzoate (9)
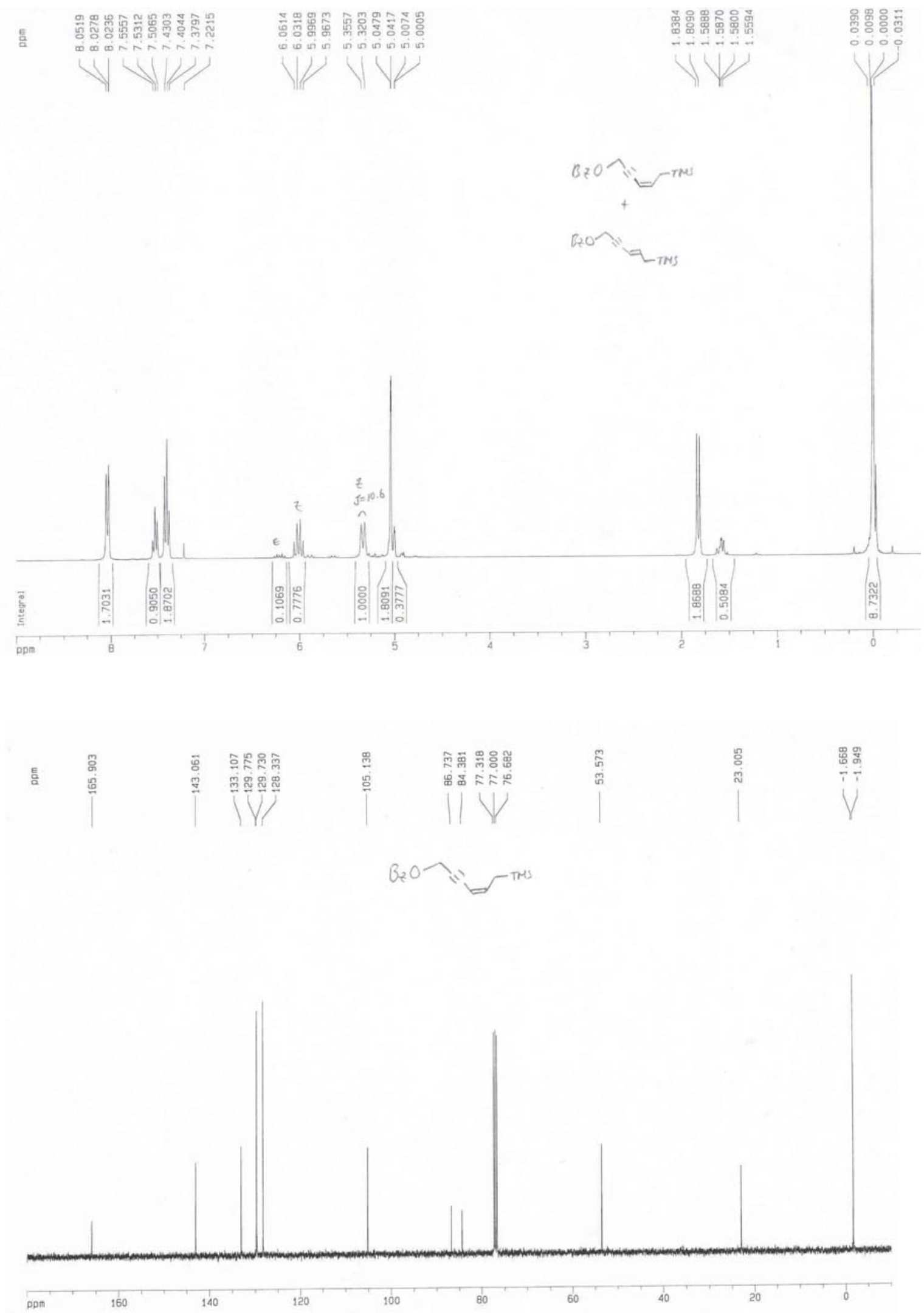


\section{[(Z)-8-Trimethylsilyl-6-octen-4-ynyl] benzoate (10)}

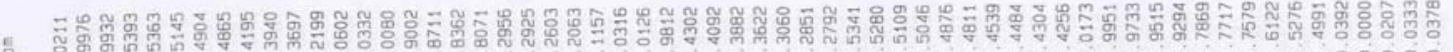

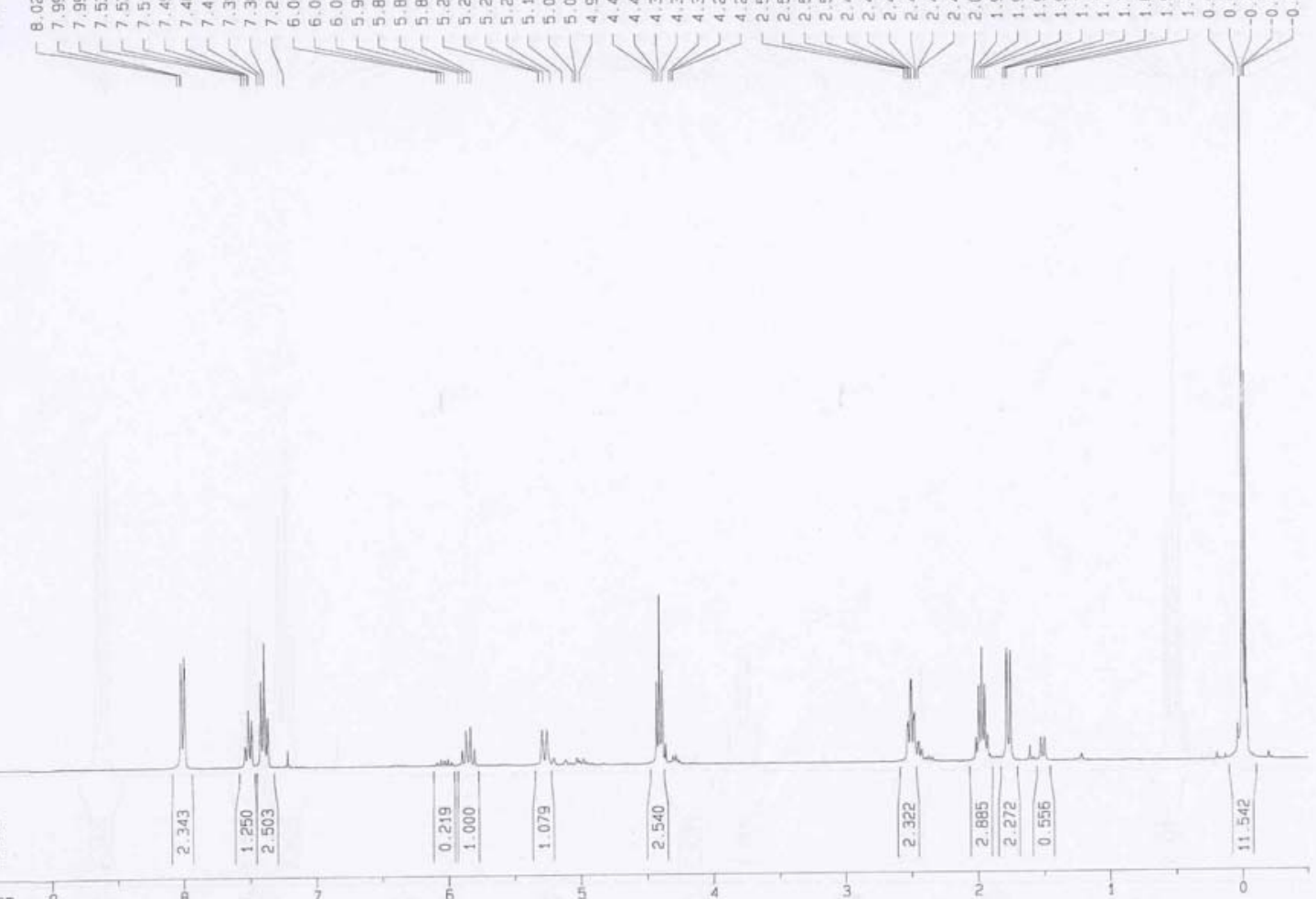

820

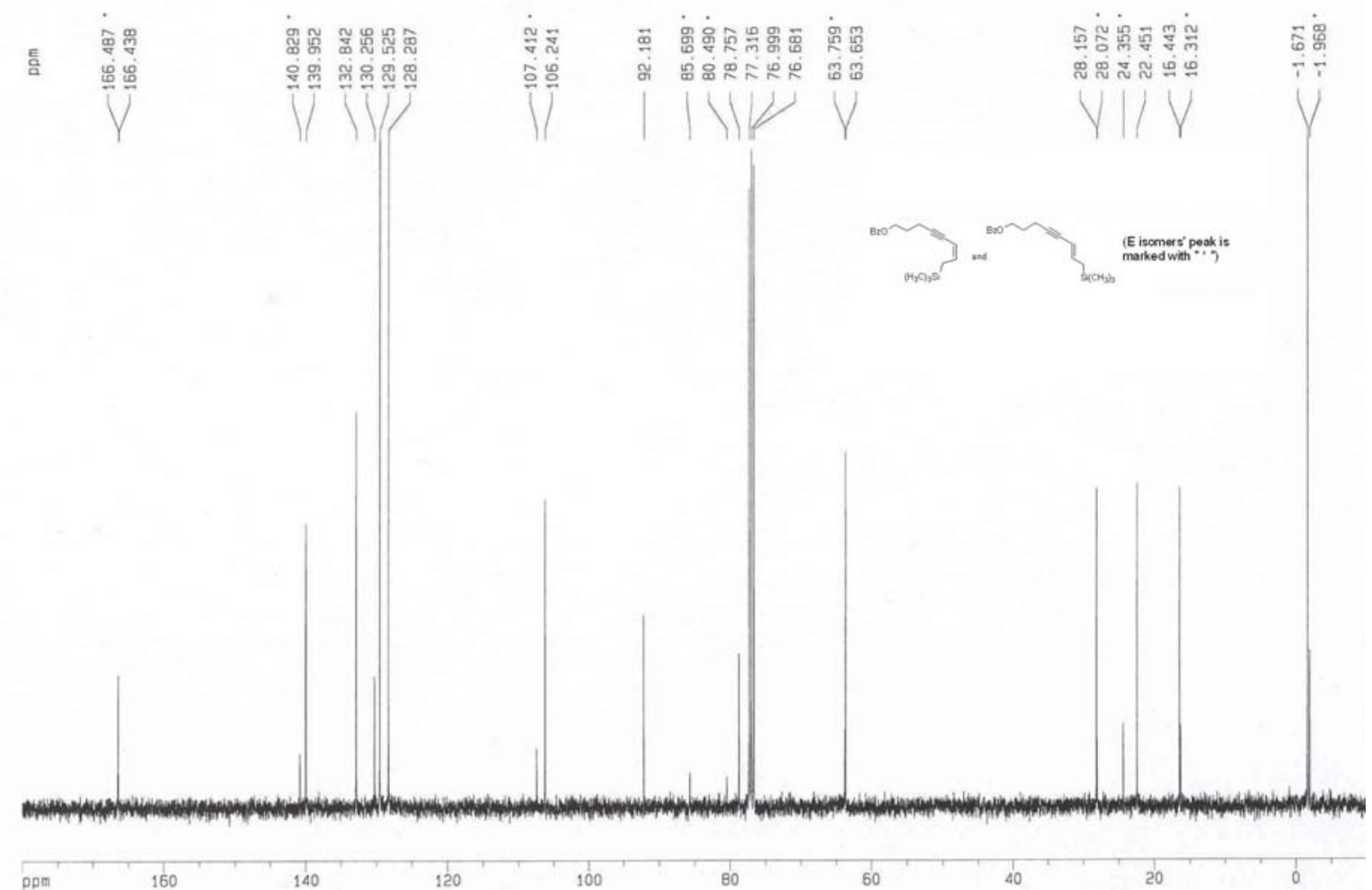


$N$-Ethyl- $N$-[(Z)-4-hepten-2-ynyl] benzamide (11)
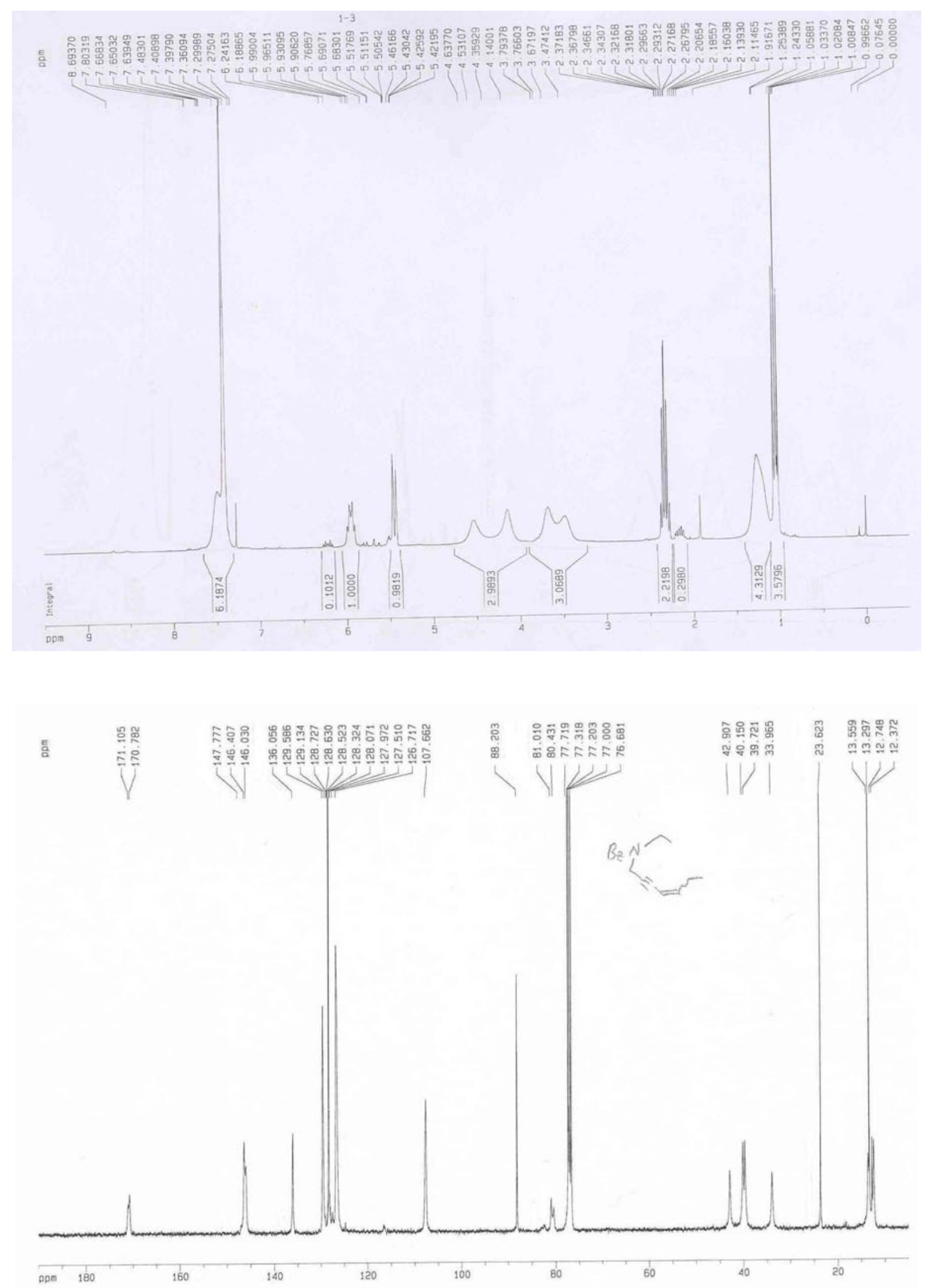
$N$-Ethyl- $N$-[(Z)-4-hepten-2-ynyl] p-toluenesulfonamide (12)

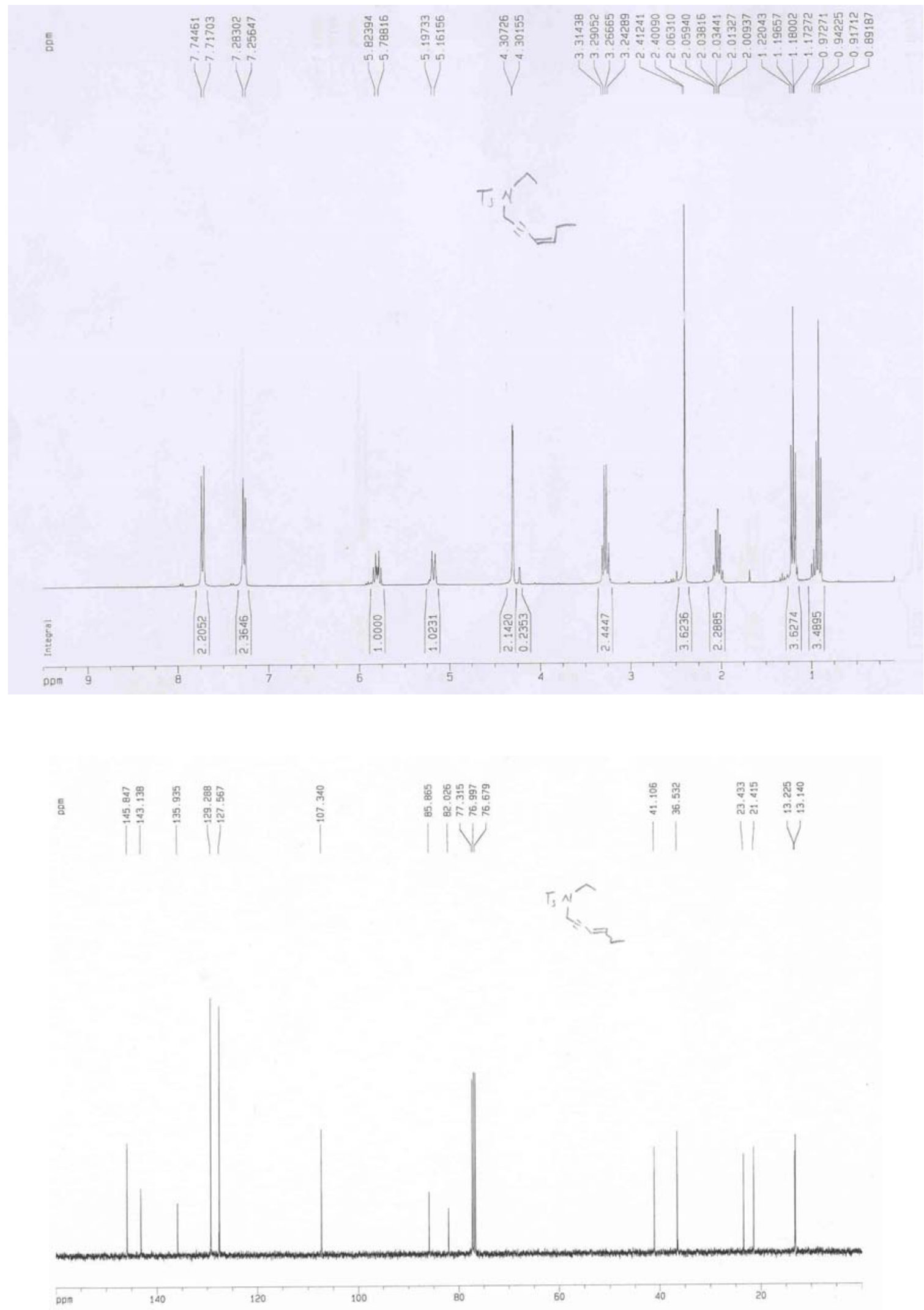


$N$-Ethyl- $N$-[6-trimethylsilyl-(Z)-4-hexen-2-ynyl] p-toluenesulfonamide (13)

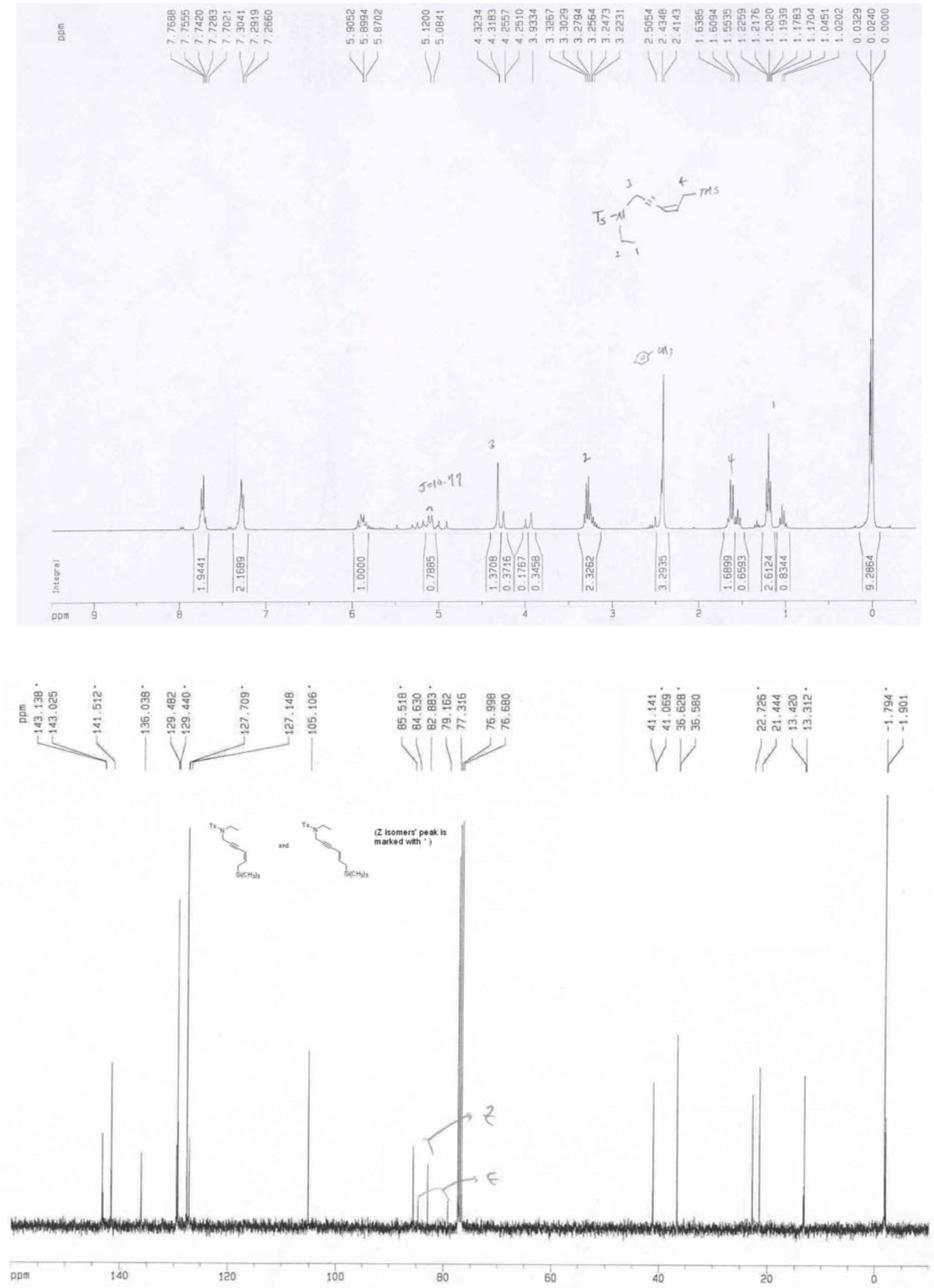


[(Z)-7-decen-5-ynyl] tosylate (14)
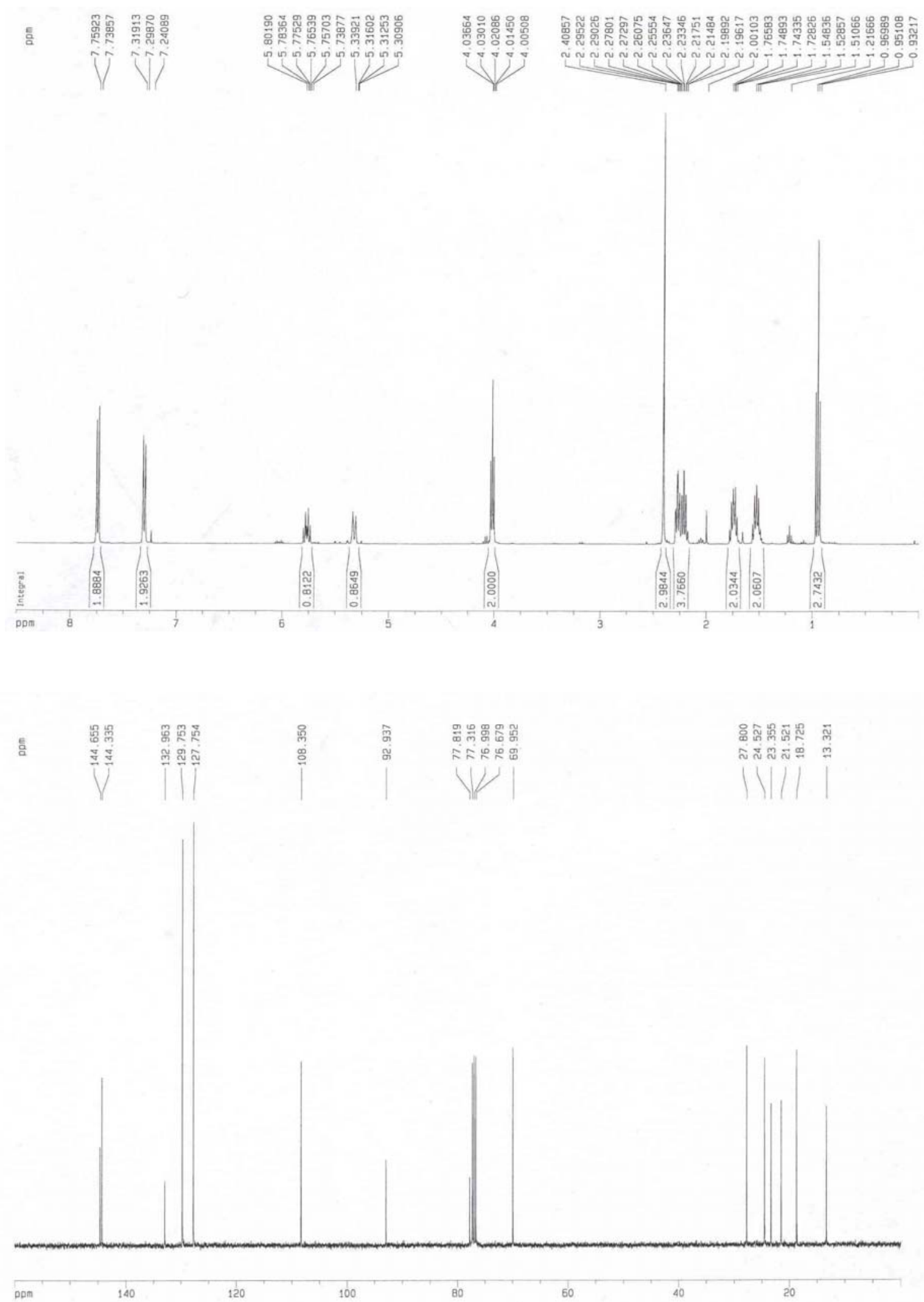


\section{[(Z)-5-octen-3-ynyl] tosylate (15)}

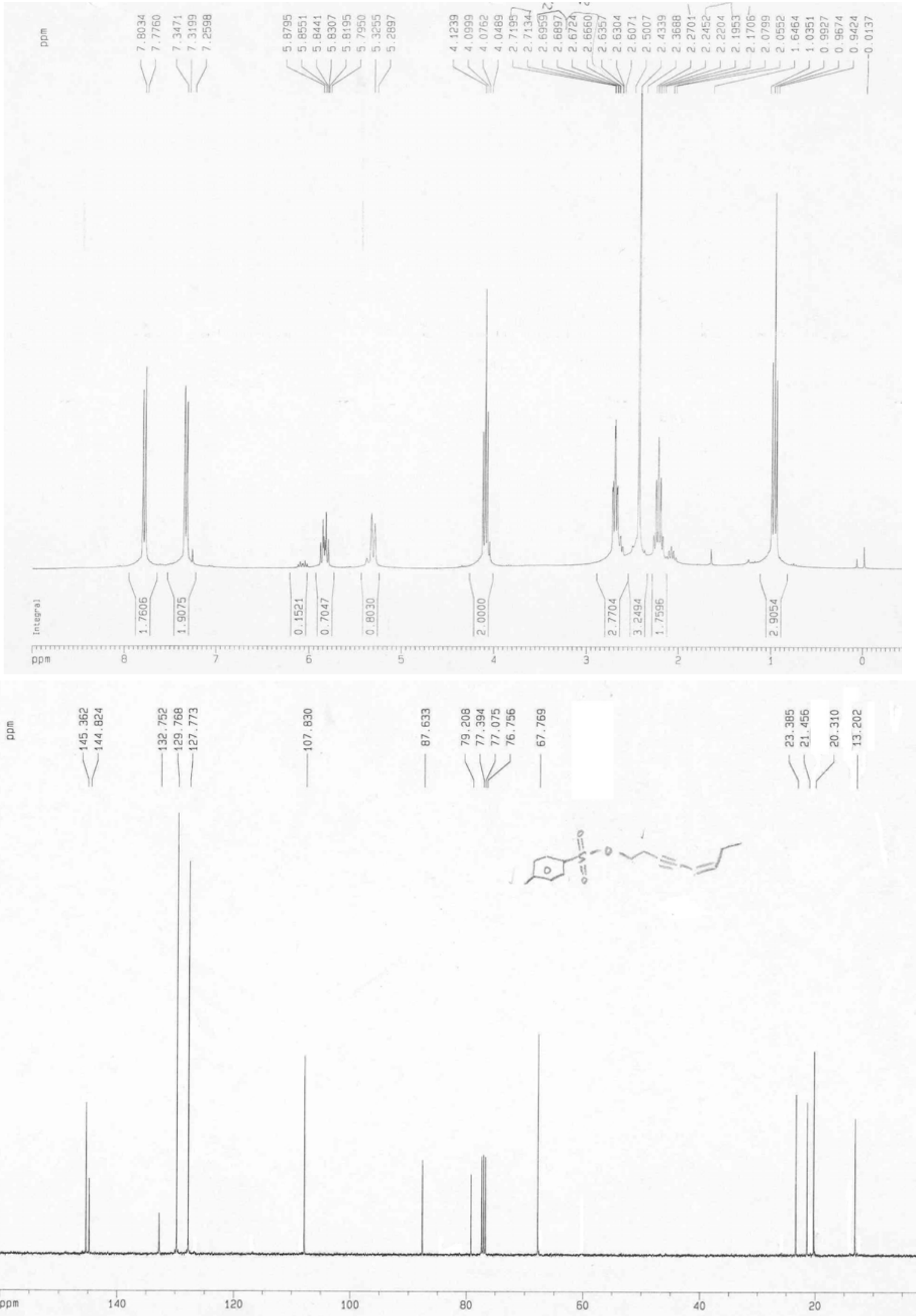




\section{Notes and References}

(1) Love, J. A.; Morgan, J. P.; Trnka, T. M.; Grubbs, R. H. Angew. Chem., Int. Ed. 2002, 41,4035 .

(2) The Gaussian 03 program used in this study: Frisch, M. J.; Trucks, G. W.; Schlegel, H. B.; Scuseria, G. E.; Robb, M. A.; Cheeseman, J. R.; Montgomery, Jr., J. A.; Vreven, T.; Kudin, K. N.; Burant, J. C.; Millam, J. M.; Iyengar, S. S.; Tomasi, J.; Barone, V.; Mennucci, B.; Cossi, M.; Scalmani, G.; Rega, N.; Petersson, G. A.; Nakatsuji, H.; Hada, M.; Ehara, M.; Toyota, K.; Fukuda, R.; Hasegawa, J.; Ishida, M.; Nakajima, T.; Honda, Y.; Kitao, O.; Nakai, H.; Klene, M.; Li, X.; Knox, J. E.; Hratchian, H. P.; Cross, J. B.; Adamo, C.; Jaramillo, J.; Gomperts, R.; Stratmann, R. E.; Yazyev, O.; Austin, A. J.; Cammi, R.; Pomelli, C.; Ochterski, J. W.; Ayala, P. Y.; Morokuma, K.; Voth, G. A.; Salvador, P.; Dannenberg, J. J.; Zakrzewski, V. G.; Dapprich, S.; Daniels, A. D.; Strain, M. C.; Farkas, O.; Malick, D. K.; Rabuck, A. D.; Raghavachari, K.; Foresman, J. B.; Ortiz, J. V.; Cui, Q.; Baboul, A. G.; Clifford, S.; Cioslowski, J.; Stefanov, B. B.; Liu, G.; Liashenko, A.; Piskorz, P.; Komaromi, I.; Martin, R. L.; Fox, D. J.; Keith, T.; Al-Laham, M. A.; Peng, C. Y.; Nanayakkara, A.; Challacombe, M.; Gill, P. M. W.; Johnson, B.; Chen, W.; Wong, M. W.; Gonzalez, C.; Pople, J. A. Gaussian 03 (Gaussian, Inc., Pittsburgh PA, 2003).

(3) Vosco, S. H.; Wilk, L.; Nusair, M. Can. J. Phys. 1980, 58, 1200.

(4) Beck, A. D. J. Chem. Phys. 1993, 98, 5648. 